ANTON BIERL

\title{
Walter Burkert - ein Religionswissenschaftler als Inspirationsquelle für eine moderne Gräzistik und kulturwissenschaftlich geprägte Literaturwissenschaft
}

\section{Einleitung}

Walter Burkert ist primär gräzistischer Religionswissenschaftler und Klassischer Philologe. ${ }^{1}$ Zugleich ist er einer der letzten Vertreter einer umfassenden Altertumswissenschaft. Sein Blick ist dabei neben spezialisierten Detailuntersuchungen oft aufs Große und Universale gerichtet, auf den Menschen schlechthin und seine psychisch-soziale Grundkonstitution. Hinter dem Philologen wird damit der Anthropologe sichtbar. Beide Tendenzen fließen in einem tiefgehenden Humanismus im wortwörtlichen Sinne zusammen. In seiner frühen Zeit ist er nach eigener Aussage geprägt worden von Otto Seel, Karl Meuli, Reinhold Merkelbach und Eric R. Dodds, die das Andere, das Irrationale und Religiöse am Griechentum betonten. In vielem erscheint er einer längst vergangenen Epoche zugehörig. Sein Werk kann sich vielleicht sogar mit deutschen altertumswissenschaftlichen Größen wie etwa Ulrich von Wilamowitz-Moellendorff oder Theodor Mommsen messen. Burkerts Schaffen gehört noch einer Zeit an, in der man sich ganz ohne Drittmittelanträge und Einschreibungen in Exzellenzinitiativen, Graduiertenschulen und Sonderforschungsbereiche individuell der Freiheit der Wissenschaft im besten Humboldtschen Sinne widmen konnte. Ähnlich wie Niklas Luhmann, der bei seiner Bielefelder Berufung gefragt wurde, was er an Ausstattung für seine Projekte benötige, nur erstaunt auf Bleistift und Zeit kam, gab er sich doch in Zürich neben seiner erfolgreichen Lehre ganz seinen ureigenen wissenschaftlichen Interessen hin. Dies alles ermöglichte wirkliche Exzellenz,

1 Direkter Schüler von Walter Burkert war ich selbst nie - dies mag auch dabei helfen, ihn hier vielleicht aus anderer Sicht und, bei allem Respekt, etwas unbefangener zu beurteilen. Nichtsdestotrotz bin ich von ihm tief geprägt, fast als wäre ich immer seinem Kreis zugehörig gewesen. Unvergesslich sind mir die erste Begegnung mit Homo Necans sowie die darauf folgende tiefe Faszination, als ich ihn zum ersten Mal in meiner Münchner Studentenzeit und dann auf der Dionysos-Konferenz in Virginia persönlich erleben durfte. 
die, wenn sie auch in Deutschland zunächst verkannt wurde, über Übersetzungen in andere Sprachen weltweit Anerkennung fand. Sein ganz eigener Ansatz ist zudem geprägt von der Herkunft und den historischen Entwicklungen, das heißt von der Nachkriegszeit und der allmählich einsetzenden geistigen Öffnung der BRD sowie von den kulturellen Umwälzungen um das Jahr 1968.

Walter Burkert ist es gelungen, über seine eigentliche Disziplin der Gräzistik hinaus eine allgemeine Bekanntheit als Geisteswissenschaftler zu erzielen. Trotz einzigartiger Gelehrsamkeit vermag er offensichtlich etwas Grundsätzliches anzusprechen, was uns alle angeht, eine verdrängte Tiefenschicht unserer anthropologischen Existenz, die im Mythos und im (in einer mehr oder minder losen Beziehung dazu verlaufenden) Ritual ihren Ausdruck sucht. Burkert geht zum "wilden Ursprung" unserer westlichen, von den Griechen so beeinflussten Zivilisation zurück. ${ }^{2}$ Im geistigen Aufbruch der frühen 1960er Jahre und auf den vorausliegenden Erfahrungen des Schreckens des letzten Jahrhunderts basierend verlässt er die eingetretenen Pfade positivistisch-historistischer oder neo-humanistischer Forschung in den Altertumswissenschaften. Auf einer synthetischen Theoriebasis versucht er dabei zu den fundamentalen Zusammenhängen menschlichen Lebens vorzudringen. Wie ein roter Faden zieht sich die Überlegung durch sein Werk, dass für die menschliche Zivilisation nicht nur die Verdrängung des angeborenen Triebs zur Aggression und Gewalt, sondern auch deren konstruktive Einbindung vonnöten ist. In der kulturellen Verarbeitung durch letztlich auf biologische Handlungsprogramme zurückführbare Riten und Mythen kann nämlich die destruktive Energie für das Zusammenleben konstruktiv umgepolt werden. Nach Burkert ist es dadurch möglich, Krisen im individuellen und gesellschaftlichen Leben erträglich zu machen und zu überwinden. In den zentralen Praktiken wie Opfer, Initiation und Fest agiert man performativ die inhärente Energie aus, um sie für die dauerhafte Kohäsion fruchtbar zu machen. Es findet also laufend eine Art Rückversicherung bei den Ursprüngen statt; in der Rückkehr zu diesen kann das Chaos bewältigt werden. Damit wird eine Begründung und Legitimierung der bestehenden Ordnung gewährleistet.

2 So der gelungene Titel, den Glenn Most (vgl. auch Most 1990) einer von ihm herausgegebenen Sammlung von einigen frühen Aufsätzen Burkerts (1990a) gab. 


\section{Arbeitsfelder}

Bereits seine in Erlangen entstandene Dissertation (Burkert 1955) behandelt ein für Burkert typisches Thema, den altgriechischen Mitleidsbegriff. In der Habilitation Weisheit und Wissenschaft (Burkert 1962a) holt er schon zu seiner charakteristischen Weite aus, indem er vorsokratisches Denken und mathematische Theorie in religiöse Kontexte setzt und dabei das wichtige, von Meuli und Dodds stammende Konzept des griechischen Schamanismus aufgreift. Der wirklich große geistige Wurf gelingt ihm nach seinen Qualifikationsarbeiten in Homo Necans (Burkert 1972), wobei die zentrale These in wichtigen Aufsätzen seiner Frühzeit, die ihn vor allem auch nach USA und England brachte, vorgezeichnet ist.

Die Bereiche seiner Wirkung sind nun schnell und gut in den von Christoph Riedweg initiierten und mit anderen Weggefährten aus Burkerts Schülerkreis herausgegebenen Kleinen Schriften erschlossen, wodurch viel in fern liegenden Sammelbänden verstreut Publiziertes leicht zugänglich ist. ${ }^{3}$ Die Themen seiner wissenschaftlichen Tätigkeit kann man grob nach den Bänden zusammenfassen in: Homerica; Orientalia; Mystica, Orphica, Pythagorica; Mythica, Ritualia; Tragica, Historica; Philosophica.

Sein Werk ist durch eine eigenartige Spannung geprägt: Neben der akribischen Analyse von Detailproblemen, die unbemerkt auch immer Fundamentales berühren, steht die explizite Behandlung des ganz Großen wie in Homo Necans. Dabei arbeitet er oft mit humanethologischen und naturwissenschaftlichen Ansätzen. Sein Ziel ist es dabei, zum Wesen des Menschen selbst vorzudringen. Obwohl er ganz im philologischen Gebiet $\mathrm{zu}$ Hause ist, gilt sein eigentliches Interesse einer neuen, am Grundsätzlichen orientierten Religionswissenschaft, die für ihn zum Kern der Gräzistik aus umfassender altertumswissenschaftlicher Perspektive wird.

In seiner religionsgeschichtlichen Forschung hat er die lange in Deutschland fortbestehende 'Kluft' zwischen Ritual und Mythos geschlossen, auf der Grundlage von Jane Harrison und den Cambridge Ritualists ein Szenario von Beziehungen zwischen den beiden Bereichen eröffnet und diese Erkenntnisse auf einige wichtige Feste appliziert. ${ }^{4}$ Seine frühen Ritualstudien kreisen in einem eigentümlich klaren und kühlen, fast

3 Bisher veröffentlicht Burkert 2001; Burkert 2003b; Burkert 2006; Burkert 2007; Burkert 2008.

4 Vgl. Most 1990, bes. 10; vgl. auch Versnel 1993, 15-88. 
unheimlichen Stil, der Erkenntnisse der Soziologie und vor allem von Siegmund Freud und Konrad Lorenz aufnimmt, um die andere, eher anstößige Seite der Griechen. Der Mensch wird an der uns nahestehenden Kultur der Hellenen in seiner aus den paläolithischen Ursprüngen herkommenden Grundlage erklärt. Seine Forschungen sind zugleich von einem aktuellen Anliegen geprägt. Immer wieder weist er auf die Risiken hin, die das moderne Individuum durch die totale Befreiung von religiösen Sinnhorizonten eingeht, weil es damit seine eigene mentale Infrastruktur verleugnet. Rituale und damit verbundene mythische Traditionen sieht Burkert als theatrale und tiefe Erfahrungsmöglichkeit, sich mit Gewalt auseinanderzusetzen, um sie so in Schranken zu verweisen. Einem 'rituellen Idiotentum' versucht er durch Aufklärung Einhalt zu gebieten, ${ }^{5}$ zumal er die Gefahren des Untergangs der Religion zu sehen glaubt. Seiner Überzeugung nach hat nämlich der auf der Jagd mit Tod, Gewalt und möglicher Selbstvernichtung konfrontierte Mensch im evolutionären Prozess Praktiken wie den Opferbrauch und andere Riten und Mythen entwickelt, welche die Funktion haben, die Aggression zu bannen und zu verarbeiten. Hinter der Fassade stupender altertumswissenschaftlicher Gelehrsamkeit scheint in Burkerts Werk immer wieder ein handfestes, ganz reales kulturelles Anliegen durch: Haben wir uns in der gegenwärtigen Moderne durch den rasanten Verlust von Ritual und Mythos zentraler Dinge beraubt? Könnten wir durch die Bekanntschaft mit unseren Wurzeln, deren Inhalte durch das Zurückdrängen des Altgriechischen in den Lehrplänen unserer Schulen leider auch immer mehr verschwinden, nicht für unser heutiges Zusammenleben Grundsätzliches lernen? Und ist dies heute nach den Epochenschwellen von 1989 und 2001 nicht nötiger denn je? Krieg, Gewalt und religiöse Fanatismen kehren nach den künstlich ruhig gestellten Jahrzehnten des Kalten Krieges plötzlich auch für uns alle spürbar zurück. Ohne kulturelles Gedächtnis, ohne Mechanismen der Verarbeitung, Verdrängung und Umformung ist die Menschheit nach Burkert vielleicht plötzlich nicht mehr fähig, mit den Szenarien des Schreckens umzugehen, da sie alles, was mit Mythos und Ritual zu tun hatte, leichtfertig als irrational-primitive Diskurse über Bord warf. Damit geht auch ein Verständnis für deren Sinn verloren. Immer wieder mahnt Burkert davor, Mythos und Ritual einfach aufzugeben. Damit ordnet sich Burkert in eine Diskussion ein, die seit der späten Aufklärung bei-

5 Vgl. u. a. Burkert 1984a, 29, 32. 
spielsweise von Friedrich Hölderlin geführt wurde und nach dem Zweiten Weltkrieg mit Max Horkheimers und Theodor W. Adornos Stichwort der "Dialektik der Aufklärung" seinen Höhepunkt erlebte. Die Darstellung der weniger "erbaulichen Aspekte", um die Burkerts Darstellungen kreisen, entschuldigt er dementsprechend in der Vorbemerkung von Homo Necans

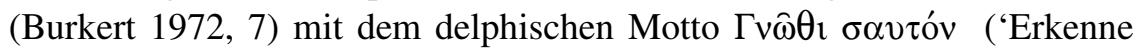
dich selbst!').

Folgende Schlüsselthemen zeichnen unter anderen sein Frühwerk aus: Mythos/Ritual; Opfer; Blut; Fest; Schuld; Heiliges/Sakralisierung; Jagd; Mysterien; Schamanen/Trickster; Tod/Todeskult/Trauer; Ursprung; Auflösung der Ordnung; Schrecken/Angst/Schauder; Sexualisierung; Leid; Gewalt/Zerreißung/Aggression.

Es liegt bei Burkert eine besondere Mischung von an Arthur Schopenhauer orientiertem säkularem Schulden- und Sündenbewusstsein, von wissenschaftlicher Durchdringung und von Glauben an Aufklärung und Fortschritt vor. Zudem ist ein Anliegen ersichtlich, das moderne Individuum mittels eines als "Schutzmacht" (Graf 1991) wirkenden religiösen Fundaments retten zu wollen. Dementsprechend herrscht in seinen Schriften ein Vokabular der Verschuldung und Entsühnung des sich am Leben vergreifenden Menschen. Hinter einem naturwissenschaftlich-atheistischen Selbstverständnis bleiben bei Burkert meist eine zutiefst christliche Sicht und ein Mitgefühl für die leidende Kreatur erkennbar. Es geht ihm kaum um das Ritual als bloße Lebenspraktik, etwa um Feier, Essen, Tanz und Heiterkeit, vielmehr interessiert er sich für heimliche Sekten, Mysten, vagabundierende Mantiker und Goeten, für Ursprünge, für menschliche Konstanten und urernste Erfahrungen. Bei allem war Burkert immer ein dezidierter Kulturwissenschaftler, noch bevor man von einer Kulturwissenschaft in der Geisteswissenschaft sprechen konnte. Durch einen bewussten Brückenschlag zur Naturwissenschaft wird bei ihm die Humanwissenschaft zu einer Kultur- und Lebenswissenschaft.

Dabei leitet Burkert in der griechischen Religionswissenschaft einen deutlichen Paradigmenwechsel ein. In radikaler Abkehr von der Tendenz, alles rituelle Handeln aus der Idee der Fruchtbarkeitsförderung in einer Ackerbaugesellschaft abzuleiten, widmet er sich dem Menschen an sich in Wiederaufnahme von Trends einer an den Theorien Émil Durkheims und Siegmund Freuds geschulten Ritualforschung der Cambridger Schule. Friedrich Nietzsche sowie Rudolf Otto und Walter F. Otto stehen zudem Pate bei dieser Denkbewegung. Ferner ist er vor allem von Konrad Lorenz 
sowie allgemein von der modernen Humanethologie und Primatenforschung tief beeinflusst. Der vorzeitliche Mensch als naked ape findet zur Jagd und macht sich damit seine natürlichen tierischen Feinde untertan. Damit beginnt der Siegeszug des hunting ape aus den Steppen Afrikas über die Welt. Die paläolithische Errungenschaft führt nach Burkert zu Kulturtechniken der Gewaltverarbeitung, zum Ritual des Opfers und zur Entwicklung anderer komplexer Ritualsequenzen, die in der Auflösung der Ordnung und ihrer Wiederherstellung das unsagbare Geschehen des gewaltsamen Tötungsprozesses verarbeiten, das die Fleischnahrung garantiert. Jagd, 'Sexualisierung' und Totenkult stehen am Anfang der menschlichen Entwicklung und werden dann über die Evolution der Gattung hinweg in Ritualisierungen wie vor allem im Opferbrauch weitergeführt, wobei Transformationen stattfinden. Das Opfer und andere verwandte Rituale werden selbst in komplexeren Stadtkulturen bewahrt, da die in theatralen Inszenierungen freiwerdende Gewalt hilft, die Gruppenkohäsion der Gesellschaft herzustellen, und als Schutz gegen ungehemmte innergemeinschaftliche Aggression fungieren kann. Da die Zeit des Getreideund Pflanzenanbaus, der den Menschen erst von der Jagd und vom Fleischgenuss unabhängig macht, entwicklungsgeschichtlich nur die letzten 10.000 Jahre eines 600.000-jährigen Zeitrahmens umfasst, hat dieser Kurzabschnitt der Menschheitsgeschichte kaum Einfluss auf die genetisch geprägte Tiefenstruktur des Homo Necans. ${ }^{6}$

Neben dem zentralen Opfer entwickelt Burkert das sozialwissenschaftlich geprägte und schon von Jane Harrison aufgenommene Paradigma der Initiation weiter, das zur selben Zeit in Italien ebenfalls von Angelo Brelich (1969) aufgegriffen wird. ${ }^{7}$ Mit den komplexeren Szenarien der Auflösung und Wiederherstellung der Ordnung, die auch im Opferkult durchgespielt werden, assoziiert Burkert die sozialen Feste der Jünglingsweihe und des Neujahrs. ${ }^{8}$ Männerbünde und weibliche Gruppen bedürfen fester Einweihungsrituale, die feierlich inszeniert werden. Damit öffnet er den Blick auch auf die wichtige weibliche Initiation, die im antiken Griechenland oft nur mehr von wenigen Mädchen repräsentativ für die ganze Altersgruppe begangen wird. Davon ausgehend kommt er zum rituellen und mythischen Pendant der Mädchentragödie, das oft als Vorspiel oder

\footnotetext{
6 Vgl. Burkert 1972, bes. 8-96; vgl. auch Burkert 1984a; Burkert 1987a.

7 Vgl. dazu Bierl 2007a, 23-24; Burkert 2004.

8 Vgl. Bierl 2007a, 28-29.
} 
Nachspiel zum Unsagbaren der totalen Gewalt eingesetzt wird. In Structure and History (Burkert 1979) dringt Burkert zu einer neuen Mythosund Ritualtheorie vor. Beide Bereiche sind ihm zufolge eng miteinander verwoben und basieren letztlich auf einem biologischen Konzept, das sich im "to get" als Grundbedürfnis manifestiert. Die These, dass jedes Ritual auf 'biologischen Spuren' gründet, wird in Creation of the Sacred (Burkert 1996a) fortentwickelt.

Der große Wurf von Homo Necans trifft bald auf eigene Bedenken und ist wohl bereits zur Zeit seiner Abfassung in vielem, gerade in der Überbetonung von Lorenz' Ansätzen, naturwissenschaftlich überholt. Im Nachwort zur zweiten Ausgabe (Burkert 1997a) relativiert Burkert angesichts der zeitlichen Differenz kräftig und nimmt dabei manches zurück. Im Bielefelder Autorenkolloquium kommt schließlich die starke Kritik an manchen biologisch-anthropologischen Annahmen ans Tageslicht und vieles wird vor und mit Burkert kontrovers diskutiert. Die in Homo Necans zugleich entworfene Ritual- und Mythengeschichte wird ferner zunehmend als Konstruktion angesehen. Burkert scheint die akribisch zusammengetragenen Quellen, die von der Früh- bis in die Spätzeit reichen, so montiert zu haben, dass sie zur modernen, von vielen Voraussetzungen und kulturellen Voreinstellungen geprägten These passen. Vor allem lässt sich dagegen fragen, wo die Kontrollinstanz über so disparate Texte unterschiedlicher Genres, Zeiten und Kontexte ist.

Bei allen Bedenken und Einschränkungen ist die weltweite Wirkung von Homo Necans, der Frühschriften, die zum Teil in der schönen Sammlung Wilder Ursprung (Burkert 1990a) vereint sind, und der genannten, weiter in Richtung Biologie zielenden Bücher zu konstatieren, vor allem über die engen Kreise der Altertumswissenschaft hinaus. Burkert wird damit zu einem modernen Kulturkritiker, ja sogar zu einer Inspirationsquelle künstlerischer Auseinandersetzung mit der Antike, sei es in der Literatur, Malerei oder Populärwissenschaft. Dies gilt vor allem für die Bühne, insbesondere für die wachsende Konjunktur der Antikeproduktionen, das heißt für die vielen Performances, Installationen und Wiederaufführungen antiker Tragödien.

Neben dieser wichtigen Rolle seines Werks als Heuristik für Altertums-, Geistes- und allgemeine Literaturwissenschaftler sowie frei schaffende Künstler ist seine Wirkung im engeren Bereich der griechischen Religion und Gräzistik zu betonen. $\mathrm{Zu}$ nennen sind hier neben den zahlreichen Aufsätzen vor allem seine weiteren Monografien. Die auf Vor- 
studien basierende griechische Religionsgeschichte Griechische Religion der archaischen und klassischen Epoche (Burkert 1977) wird zum Standardwerk, das sämtliche neuen Paradigmen diskutierfähig macht und den bis dahin üblichen Rahmen wesentlich erweitert. Alles, was man als Interessierter schon immer wissen wollte, wird hier sehr präzise angesprochen: die Prähistorie und die minoisch-mykenische Vorgeschichte, zahlreiche Einzelrituale und Heiligtümer, die Götter, der Totenkult, die Polis und der Polytheismus, Feste und Festrhythmen, das Mysterienwesen und die Verwandlung in eine philosophische Religion. Nie hat man den Eindruck eines bloßen Handbuchs, sondern immer scheint die eigene und so anregende Burkertsche Handschrift durch. Im Jahr 1984 legt er Die orientalisierende Epoche in der griechischen Religion und Literatur (Burkert 1984b) vor, wo er wie Martin West und andere die Verbindung der griechischen Welt zu östlichen Hochkulturen aufzeigt. In Ancient Mystery Cults (Burkert 1987b) untersucht er die bekannten unterschiedlichen Mysterienkulte, die er bewusst nicht, wie bis dato üblich, als späte Mysterienreligionen bezeichnet, systematisch nach Gesichtspunkten. So geht er beispielsweise darin der Frage nach, wie mit Mysterienkulten persönliche Bedürfnisse im Leben und Jenseits befriedigt werden können, welche Organisationsstrukturen und theologischen Inhalte allen gemeinsam sind und welche außergewöhnliche Erfahrung in ihnen gemacht werden kann. Eleusis, das schon eine eigenwillige Interpretation in Homo Necans (Burkert 1972, 275-327) erfuhr, und orphisch-bakchische Inhalte, die durch Goldplättchenfunde auf eine neue Grundlage gestellt wurden, spielen darin eine besondere Rolle. Da Omero ai Magi (Burkert 1999a), wie vieles aus Vortragsreihen hervorgegangen - hier spezifisch aus im Jahre 1996 in Venedig gehaltenen Vorlesungen -, im Deutschen in erweiterter Form erschienen als Die Griechen und der Orient (Burkert 2003a), ist das bisher letzte Buch. Hier kommen unterschiedlichste Interessen zusammen, wie das für Alphabetisierung und Schriftkultur, für Homer als Dichter der orientalisierenden Epoche, für ostwestliche Weisheitsliteratur und Kosmogonie als Grundlage vorsokratischer Philosophie, für Orpheus und die Orphik, für neue Quellen, insbesondere für den Derveni-Papyrus, und für die persischen Magier. Klassisches Altertum und antikes Christentum (Burkert 1996b) fasst eine Jenaer Vortragsreihe zusammen, in der es dem Religionswissenschaftler vor allem um das Verhältnis von Antike zum Christentum der Spätantike, um Gnostik, Plutarch, Platon und Paulus sowie um den Manicheismus geht. 


\section{Wirkung als Gräzist auf dem Gebiet der Literatur}

Burkerts Interesse gilt hauptsächlich der Erforschung der griechischen Religion. Selbst darin geht er häufig dem Obskuren, dem Abstrusen und Anstößigen nach, indem er das Magische, das Goetische, das Mysteriöse, das Fremde und auch das Dionysische immer wieder zum Gegenstand wählt. Falls er sich zu den Göttern äußert, haben Apollon und Dionysos eine bevorzugte Stellung inne. ${ }^{9}$ Angesichts Burkerts Konzentration auf Ernstes und Schreckliches, Blut, Wahnsinn, Ekel, Schauder und Opfer steht bei ihm vergleichsweise wenig zu heiteren Gesichtspunkten der griechischen Religion, zu ausgelassenen Feiern, zum Lachen, Scherz und Tanz. Wenn er überhaupt griechische Literatur interpretiert, dient sie ihm meist eher als Quellenmaterial zur Erklärung religionsgeschichtlicher Fragen als dass es ihm um literarische Auslegung im eigentlichen Sinne ginge. Nur in den seltensten Fällen tritt der Klassische Philologe als Interpret und Literaturwissenschaftler der großen griechischen Texte auf. Auf überraschende Weise macht er auch kaum den entscheidenden Schritt, seine faszinierenden Erkenntnisse und Modelle, die er zum Teil aus literarischen Quellen ableitet, als Konstruktionsprinzip und Basis der antiken Literatur selbst zu sehen. Oft erweckt es den Anschein, als schrecke Burkert vor einer solchen konsequenten mythisch-rituellen Lektüre zurück. Es stellt sich die Frage, ob der Antiklassiker hier doch noch zu sehr vom Klassizismus geprägt ist.

Ich komme später auf diese neuere Erkenntnis einer mythisch-rituellen Poetik zurück, die Burkert selbst noch nicht ausformuliert. Die Generation seiner Schülerinnen und Schüler konnte hier anschließen. Erneut ist festzuhalten: Auch auf diesem Gebiet lässt sich Burkerts Werk hervorragend als heuristisches Instrumentarium verwenden. Im Bereich der literarischen Interpretation in diesem Sinne bieten sich zwei Wege an. Entweder man lässt sich von ganzen Burkertschen Theorieblöcken anregen oder man appliziert bestimmte, von ihm herausgearbeitete religionswissenschaftliche Detailkenntnisse. Für den ersten Fall muss man freilich Vorsicht walten lassen. Heute sieht man sehr deutlich die stark universalistischen und reduktionistischen Tendenzen in Burkerts Theoriegebäude, das in manchem nicht mehr dem Stand der Erkenntnis entspricht. Ritual wird von ihm als zu statisch und unveränderlich angesehen, während man heute

9 Zu Apollon vgl. u. a. Burkert 1975. 
gerade die Dynamik und Adaptionsfähigkeiten des Rituals erkennt. ${ }^{10}$ Literatur ist zudem nie nur ein Spiegel von lebensweltlichen Praktiken, sondern in mehr oder minderer Autonomie kann sie im freien Spiel von Variation, Kombination und Wiederholung Einzelbestandteile von Ritualsequenzen zu einem eigenen ästhetischen Produkt zusammensetzen.

Auf dem Felde der gräzistischen Literatur beschäftigt sich Burkert einerseits mit den kanonischen Texten, andererseits auch mit Randgebieten. Auffallend gewirkt hat er in der Erforschung des Homerischen Epos, der Vorsokratik und der Tragödie. Gehen wir die Gebiete im zeitlichen Verlauf anhand ausgewählter Beiträge durch:

\section{III.1 Homer}

Zunächst bewegt er sich noch ganz in den traditionellen Bahnen der für Deutschland typischen Auseinandersetzung zwischen Analyse und Unitarismus. Ohne auf die Mündlichkeitsforschungen von Milman Parry (u. a. 1928; 1971) und Albert Lord (1960) einzugehen, interpretiert Burkert (1960) das Lied von Ares und Aphrodite im achten Gesang der Odyssee als literarische Referenz auf die Hephaistosszene in Ilias 1 und den Betrug des Zeus in Ilias 14, und bezieht auch hier religionswissenschaftliche Erkenntnisse ein. Später wird Homer das Territorium schlechthin, an dem er den orientalisierenden Einfluss, den Austausch zwischen Ost und West und die Abhängigkeit Homers von vorderorientalischen Modellen zeigt. Ferner behandelt er immer wieder Religiöses in Detailstudien. Zudem geht es ihm bei Homer zunehmend um die Einführung und den Reflex der Alphabetisierung sowie um die Datierung, die er ins frühe sechste Jahrhundert v. Chr. und damit relativ spät ansetzt. Das hunderttorige Theben in der Ilias bezieht Burkert (1976) auf die Eroberung durch Assurbanipal im Jahre 663 v. Chr., was zugleich als terminus post quem des Epos dienen kann. Die Tradition der Sieben gegen Theben betrachtet er ferner als Reflex eines babylonischen magischen Heilrituals zur Vertreibung des Bösen, nicht wie üblich als Spiegel realer geschichtlicher Ereignisse (Burkert 1981). Phantastische Tiefenschichten der Odyssee deutet er in Homo Necans (Burkert 1972, 148-152 und 178-181) an, die er nie detailliert am literarischen Text ausführt. Für die Kannibalengeschichte des

10 Vgl. den SFB 619 "Ritualdynamik" zu Heidelberg und Harth/Schenk 2004. Vgl. auch den Beitrag von Eveline Krummen in diesem Band. 
berühmten Polyphem-Abenteuers postuliert er das Substrat der Werwolfgeschichte und des Menschenopfers. Der später geschlachtete Widder, der Odysseus vor dem Menschenfresser rettet, indem der Held sich am Fell unter dem Bauch des Tieres festkrallt, ist nach Burkert das Opfer, mit dem sich der Mensch identifiziert. Als nichtgriechische Figur reicht OdysseusOlytteus bis in Vorzeitliches zurück. Auf der Grundlage eines Scholions zu Apollonios Rhodios 1.917 bringt Burkert Odysseus mit den Mysterien von Samothrake zusammen, wo sich auch alles um ein Widderopfer drehte. Die Bekleidung mit einem Wollband als Symbol der Errettung vor dem Ertrinken assoziiert er mit dem Schleier, den ihm in größter Seenot Ino-Leukothea in der Odyssee zuwirft. Mythen verdeutlichen wie immer die rituelle Grundlage. Die Rückkehr des Dardanos, des Gründers von Troia, auf einem Floß zur Zeit der Ur-Sintflut setzt er mit Odysseus' Floßfahrt von Kalypsos Heimat Ogygia in Parallele. Der Name dieser Insel steht nach Burkert mit Ogygos, dem Urkönig Böotiens, in Verbindung, der ebenfalls mit einer Urflut in Zusammenhang gebracht wird. Diese Deutung findet Unterstützung im böotischen Kabirenheiligtum. Unter den grotesken Figuren findet sich immer wieder "Olyteus" auf dem Floß treibend in einer Sintflut, welche die Inversion der Ordnung bedeutet; mit einem Filz-Pilos bekleidet erscheint er fast als Kabire, der in die Wolle seines Widderopfers gehüllt ist. Nach dem Werwolfschema isst er nämlich vom Menschenopfer - die Blendung des Ogers mit einem feuergehärteten Speer verweist auf uralte Härtetechnik vor der Erfindung des Eisens -, dann flieht er unter dem Widder beziehungsweise im Widderfell, was die Identifizierung mit dem geopferten Tier versinnbildlicht. Neun Jahre muss er auf Irrfahrten, bis er am Apollonfest nach Ithaka zurückkehrt. Die Auflösung der Ordnung an den Skira spiegelt sich in der Sagenversion wider, nach der Troia ebenfalls am 12. Skirophorion erobert wurde. Das troianische Pferd symbolisiert nach Burkert ein uraltes Pferdeopfer als Auflösungsopfer. Das Detail der berühmten Bogenprobe mit dem Schuss durch die zwölf aufgestellten Doppeläxte bringt er in einem anderen Beitrag (Burkert 1973) mit der Angabe zusammen, dass ägyptische Pharaonen des Neuen Reichs den Pfeil durch Kupferplättchen jagten. Anderswo erklärt Burkert (1984c) Hermes im Homerischen Hermeshymnos, der sich am Opfer vergreift, mit dem Modell des Tricksters. 


\section{III.2 Vorsokratik}

Burkert (1962a; 1962b) bringt zudem den Goes in Verbindung mit den großen weisen Männern der Vorsokratik. Weisheit und Wissenschaft - so auch der Titel des gleichnamigen Buchs (Burkert 1962a) -, die im engen Austausch des Ostens mit dem Westen entwickelt werden, stehen als Wegbereiter der griechischen Philosophie im Zentrum seiner diesbezüglichen Beschäftigung. Gurus und Spezialisten spielen hier eine große Rolle. ${ }^{11}$ Seit seiner Habilitation fasziniert Burkert die Thematik der Pythagoreer, der frühen Mathematiker, die mit Orphischem und Mysteriensekten in engem Zusammenhang stehen. Bei der Aufbereitung und Analyse der spektakulären Neufunde in diesem Bereich, besonders bei der textlichen Rekonstruktion und Interpretation der orphischen Goldblättchen und des 1962 gefundenen Derveni-Papyrus, hat Burkert (u. a. 1968; 1997b) an vorderster Front mitgewirkt. Auch die Kosmogonie als Gattung möglicher Ritualtexte wurde von Burkert (u. a. 1999b) wiederholt in den Blick genommen.

\section{III.3 Platon}

Bei diesem zentralen Philosophen geht es ihm weniger um die Einzelanalyse von Dialogen, die zum Teil wiederum auf mythisch-rituellen Elementen basieren, als um die Überlieferung, den Erkenntniswert und den Beitrag für die weitere Entwicklung der griechischen Religion, die sich mit Platon zu einer nahezu philosophischen Religion wandelt. ${ }^{12}$

\section{III.4 Herodot}

$\mathrm{Zu}$ diesem Historiker publiziert Burkert häufiger, da er wohl dessen Verankerung in mythisch-rituellen Schemata erkennt. ${ }^{13}$ Doch blickt er auch hier mehr auf Details als auf das Ganze. Demaratos' fabulöse Abstammungsgeschichte (6.61-69), die sich am Amphitryonmythos anlehnt, betrachtet er beispielsweise mehr aus der Perspektive persischer Propaganda als in Erzählzusammenhängen (Burkert 1965). Wie bei mehreren anderen Figuren könnte man auch die Beschreibung des Demaratos deutlicher als

11 Vgl. Albert Henrichs in diesem Band.

12 Vgl. u. a. Burkert 1993a und das eigene Kapitel in Burkert 1977, 452-495, bes. 473-495.

13 Vgl. u. a. Burkert 1965; Burkert 1985b. 
Leistung der symbolisierenden Figurenzeichnung beurteilen, die im Dienste der Gesamtaussage steht. Harpagos' schreckliches Schicksal (1.108119) wird im Kontext der Atreusmähler relativ kurz in Homo Necans behandelt. Implizit spricht Burkert $(1972,125)$ davon, dass Herodot diese Zeichnung zur Markierung des Übergangs der herrschenden Völker von den Medern zu den Persern einsetzt, auch wenn er dies in der Anbindung an das Werwolfschema so gar nicht explizit macht. Wie gesagt fällt auf, dass Burkert hier meist in Einzelandeutungen stehen bleibt, die zu ausführlicheren Interpretationen auf einer mythisch-rituellen Grundlage anregen.

\section{III.5 Tragödie}

Die Tragödie ist seit dem berühmten Aufsatz zum Opferritual (1966b) die Gattung schlechthin, anhand derer Burkert der Thematik des unsagbaren und brutalen Opfers auf den Grund geht. Das Entsetzen über das schreckliche Geschehen findet sich künstlerisch verarbeitet in diesem ernsten Genre, das nach seiner Interpretation auf eine antike Tradition hin als "Gesang beim Bocksopfer" bzw. "um den Preis eines Bockes" aufgeführt wird. ${ }^{14}$ Das Opfer ist zudem für das weitere Verständnis der Gattung konstitutiv. Auch hier eignet sich Burkerts Werk erneut deutlich als heuristisches Instrumentarium nicht nur für antike, sondern auch für moderne Tragödien; ${ }^{15}$ kaum leistet er dies für die Antike in literarischen Analysen selbst. Doch im bereits genannten Artikel (Burkert 1966b, 117-121) kommt er ausnahmsweise auf einige Beispiele zu sprechen. Er geht nach Erwähnung offensichtlicher Fälle wie von Euripides' Iphigenie in Aulis, Iphigenie auf Tauris, Bakchen, Herakliden, Hekuba, Phoinissen, Erechtheus und Phrixos deutlicher auf die Trachinierinnen des Sophokles, auf Euripides' Medea und den Agamemnon des Aischylos ein. Nur in einem einzigen späteren Aufsatz (Burkert 1985a) kommt er nochmals darauf zurück und analysiert fast schon im Sinne einer mythisch-rituellen Poetik das Entsühnungsritual in Sophokles' Oidipus auf Kolonos, erneut die Trachinierinnen mitsamt der "Verkettung von Schuld, Sühne und Rache" (ebd. 85) und dem entscheidenden Stieropfer am Kap Kenaion sowie die Perversion des Opfers in der Antigone. In einem anderen einflussreichen

14 Burkert 1966b, dt. Zitat in Burkert 1990a, 14.

15 Vgl. Braungart 2007, bes. 440 zur Heuristik. 
Artikel spricht Burkert (1974) von der "Absurdität der Gewalt" im Orestes, die Euripides seiner Meinung nach offenlegt. Das Motiv der Reinigung wird in konkreten Ritualen von Selinus ausgemacht (Burkert 1999c). Reflexen attischer Feste geht Burkert (1993b) in Euripides' Iphigenie in Aulis nach, wo er unter anderem das berühmte Apobates-Ritual der Panathenäen nachweist. In anderen Aufsätzen behandelt er zum Teil philologische Fragen wie die Datierung der Elektra des Euripides (Burkert 1990b) und die Bedeutung des Orakels im Oidipus Tyrannos des Sophokles (Burkert 1991; Burkert 2000).

Hesiod, die Lyrik im engeren Sinne, die Komödie und das Satyrspiel, die hellenistische Poesie und der griechische Roman sind literaturgeschichtliche Felder, die Burkert nicht ausführlich behandelt, auch wenn er vereinzelt $\mathrm{zu}$ diesen Autoren und Gattungen beiläufige Bemerkungen macht. Beim Roman hat es fast den Anschein, als wolle er sich nicht auf das Territorium seines verehrten Vorbilds Reinhold Merkelbach begeben, der den Roman als Mysterientext liest. ${ }^{16}$ Obwohl sich das Genre trefflich zur Demonstration der Mädchentragödie und ihrer literarischen Verarbeitung eignet, geht Burkert (1996a, 69-79) nur einmal kürzer auf Apuleius und die darin eingelegte Erzählung von Amor und Psyche ein.

\section{Homo Necans, die frühen Aufsätze und das Tragische}

Homo Necans und die frühen Aufsätze, insbesondere derjenige zum tragischen Opfer (Burkert 1966b), sind in Bezug auf eine Anwendung auf die Literatur wohl am interessantesten. Es geht, wie gesagt, hier um eine gröBere anthropologische und universalistische Synthese. Literarische Texte fließen wiederholt in ein Konvolut von anderen Quellen ein. Das bis auf die altsteinzeitliche Periode zugreifende Modell einer Auflösung und Wiederherstellung von Ordnung im Opfer ist sehr nützlich, in seiner übergroßen Allgemeinheit allerdings nur in Bezug auf einzelne Riteme und mythische Muster wiederum fruchtbar auf Literatur anwendbar. Hierbei gelingt Burkert ein Paradigmenwechsel im Sinne der Soziologie und

16 Vgl. Merkelbach 1962; Merkelbach 1988; Merkelbach 1995; dazu positiv Burkert 1987b, 66-67. Zu einer Zurückweisung der Mysterienthese vgl. Bierl 2007b, bes. 250, 258-265 und zum Roman s. unten V.6. 
Psychologie von Gesellschaft, Gruppe und Individuum. Höchst produktiv ist die Einführung des Paradigmas der Initiation in Verbindung mit dem Jahresfest und Königsritual. Ursprünglich hätte Homo Necans eigentlich ein Buch über die Initiation werden sollen oder eine allgemeine, heortologische Religionsgeschichte. Herausgekommen ist ein viel eigenwilligeres Buch zum Opfer in der griechischen und menschlichen Kultur, wobei in der Einzelanalyse diese anderen Gesichtspunkte allesamt souverän eingeflochten werden. Nahezu jedes Detail, selbst bestimmte Festabläufe wie die idiosynkratische Rekonstruktion der Anthesterien und der Mysterien von Eleusis, wird dem Schema eines Aufbrechens der Ordnung im Opfer und der darauf folgenden Opferrestitution unterworfen. Heimlicher Akteur hinter allem wird Dionysos, ${ }^{17}$ der für Burkert interessanterweise in Aufnahme der damaligen communis opinio zu einem urtümlicheren griechischen Zustand hinzutritt und alles in seinem Sinne dramatisch auflädt. ${ }^{18}$ Dionysos wird für ihn zur später hinzugekommenen Chiffre eines Metaablaufs im Sinne eines Lebensdramas in einer deutlich von Nietzsche und Walter F. Otto geprägten Lesart. Mögliche Widersprüche, die dem pattern entgegenstehen, werden als Umschlagen und gegenseitiges Verschränken von Polaritäten wegdiskutiert. Spannungen zwischen Gegensätzen lösen sich ineinander auf, indem beide Seiten der in der Narration auseinander tretenden Pole im Dionysischen zusammenfallen. ${ }^{19}$ Besonders relevant wird dies im großen dritten Kapitel "Auflösung und Neujahrsfest", in dem das Hereinbrechen der Gewalt im Opfer und die Wiederherstellung des Kosmos-Zustandes an Stadtfesten durchgespielt werden. Bei den Agrionia fließen Ritual und Mythos ineinander und sie erhalten gewissermaßen einen dionysischen Anstrich. Dabei werden die Erzählungen der Proitiden und Minyaden in Parallele gesetzt und die Geschichten von Tereus und der Nachtigall, von Antiope und Epopeus sowie von den lemnischen Frauen hinzugestellt. Das Kochen im Kessel assoziiert Burkert mit der berühmten Geschichte des Todes des Dionysosknäblein, der von den Titanen getötet und nach Kochen und Braten verspeist wird. Ebenso steht hinter den Atreusmählern im Dreifußkessel implizit Dionysos, besonders

17 Vgl. auch Renate Schlesier in diesem Band.

18 Zum Vordringen des Dionysos vgl. Burkert 1972, 205-206, 208, 218, 235. Zur alten communis opinio vgl. Bierl 1991, 19 mit der Zurückweisung. Dionysos ist ein uralter Polisgott; vgl. Bierl 1991, 45-54 und passim; Isler-Kerényi 2001.

19 Vgl. u. a. Burkert 1972, 108, 153, 196-197. 
in Delphi. ${ }^{20}$ Das Schema erinnert in manchem an strukturalistische Erzählung, bevor Burkert diese Theorie dann in Structure and History (1979) explizit aufgreift. ${ }^{21}$ Elemente werden variiert, vertauscht und neu kombiniert, Gottheiten als Motivationsfaktoren ausgetauscht. Gewissermaßen als 'Übergottheit' bringt Burkert immer wieder den tragischen Dionysos ins Spiel. Das konkrete Tieropfer wird damit grundsätzlich zum Zeichen der Auflösung: Die dionysische Gewalt bricht in die Gesellschaft hinein und schließlich wird über Ritual das Opfer wiederhergestellt und die Ordnung erneut aufgebaut. Das inhärent Theatrale und Dramatische dieser Rituale und der sie begleitenden Erzählungen wird als symbolisches drama gedeutet. ${ }^{22}$

Daher möchte ich als These formulieren: Der ganze Gedankengang von Homo Necans sowie sein implizites pattern beruht auf dionysischen und tragischen Vorstellungen, die mehr oder minder bewusst einer Nietzscheschen Lesart unterzogen sind. Alles wirkt bei genauerem Hinsehen dramatisch und tragisch ante festum. Außerdem erkennt man in Burkerts nüchterner und doch fast poetischer Darstellung durch die Betonung des Theatralen und Dramatischen ein Bewusstsein über die Performativität des Rituals. Und letztlich beruht das gesamte Konstrukt von Homo Necans auf der theoretischen Grundlage des berühmten Bocksopfer-Aufsatzes, der Tragödie als "Gesang beim Bocksopfer" bzw. "um den Preis eines Bockes" deutet. ${ }^{23}$ Die attische Tragödie wird damit für Burkert in gewisser Weise nur zu einer kulturellen Sonderleistung, die das dramatisch-tragische Potential des Mythos und Rituals dieses ganzen Komplexes als theatrales Produkt im Kontext eines Festes vor der Polis ausstellt, gewissermaßen eine kulturelle Wucherung eines uralten Schemas in neuen kulturellen Zusammenhängen. Hinter der Verschiebungsarbeit lauert das altsteinzeitliche Substrat, der ursprüngliche Kern des Tieropfers, das dem hochentwickelten attischen Theater wie dem Lebensdrama im Allgemeinen zu Grunde liegt. Das bedeutet in Burkerts Logik: Alle rituellen Gelegenheiten - seien es die eleusinischen Mysterien, die Anthesterien, der Zyklus von den Buphonia über die Skira bis zu den Panathenäen, die Agrionia, oder andere Stadtfeste, an denen Opfer stattfinden - und zahl-

20 Vgl. Burkert 1972, 140-142; zum zerrissenen Dionysos vgl. Burkert 1972, 249, 257.

21 Vgl. Riu 1999 mit der Rezension von Bierl 2002b.

22 Vgl. u. a. Burkert 1972, 34, 35, 43, 52.

23 Burkert 1966b, dt. Zitat in Burkert 1990a, 14. 
lose mythische Reflexe, die den nämlichen Komplex im Öffentlichen wie im Privaten, d. h. im Bruch der Ehe, durchspielen, greifen ein uraltes, im paläolithischen Jägerritual gründendes Muster auf, das in sich ein großes narratives und theatrales Potential birgt. Die Auflösung der Ordnung findet ihre späte göttliche Konkretisation in Dionysos, zu dessen Ehre dann die Tragödie aufgeführt wird. Der Gott des Weins, der Mania, der Frauen, der Maske und des Lebens nach dem Tode ist dafür prädestiniert. Und im Opfer des Bocks, der den Weinstock bedroht, wird die ganze Gewalt gewissermaßen hereingelassen. Das Opfer ist also nach Burkert die eigentliche Basis jeglicher tragischer Produktion. Hinter seiner Behandlung der Proitiden und Minyaden im Homo Necans (Burkert 1972, 189-200) steht implizit das Modell der Euripideischen Bakchen. Denn hier wird ebenfalls von Dionysos eine sich ihm widersetzende Gruppe von Frauen von ihren Webstühlen in die Bergwelt und in den Wahnsinn getrieben. Die Anhängerinnen des Gottes unterteilen sich außerdem in vergleichbarer Weise in positive asiatische Bakchen und negative thebanische Mänaden. Die beiden Gruppen treten auseinander und stehen sich feindlich gegenüber, genauso wie Dionysos seinem Alter Ego Pentheus. Die Verfolgung wie auch das grausame Opfer am Widersacher, der damit ein bakchisches Ritual empfängt, gehören in der Logik Burkerts und anderer zu der für Dionysos charakteristischen Verschränkung gegenstrebig verschränkter Polaritäten. In der Zusammensetzung des zerrissenen Leichnams, einer Szene, die leider nur in einer Textlücke über den byzantinischen Christus patiens rekonstruiert werden kann, hätten wir demnach die Restitution des zum Tieropfer verschobenen Menschenopfers. Auch die Geschichte der Antiope wird dann erst in der Euripideischen Fassung zu einem dionysischen Drama des Lebens angesichts des Todes und der Gewalt. ${ }^{24}$

Hinter diesem impliziten Dionysos-Drama steht ein Gewebe von Ritualen und Mythen, die aufeinander bezogen bleiben. Das Opfer ist demnach für Burkert das Metaritual schlechthin, das Erzählungen und die Tragödie generiert. Daneben stehen die anderen Paradigmen, die Initiation, das Jahresfest zu Neujahr, das Königsritual, das Ausnahmefest und das Pharmakosritual. Lediglich vom Vegetationsfest will Burkert nichts wissen. Eingebettet sind der Agon, der im Todeskampf und im Wettkampf nach der Wiederherstellung seinen Ausgang hat, die Jagd in umschlagenden

24 Burkert 1972, 207-209. 
Bildern des Jägers, der zum Gejagten wird, Krieg, Klage, 'Sexualisierung', Mänadentum, Mysterien, Orphisches, die Aktaionsage und ZerreiBungsmotive, sowie die Trennung der Geschlechter und ihre Wiederzusammenführung. Wir werden sehen, wie andere Forscher nach Burkert diese Übertragung auf die literarische Gattung der Tragödie, insbesondere auf die Bakchen, vollzogen.

Nach dem Tragödienbeitrag (Burkert 1966b, 117-121) nimmt Burkert außer in einem weiteren Aufsatz (Burkert 1985a) keine weiteren Applikationen auf Tragödien und andere literarische Texte mehr vor. Vielleicht liegt der Grund darin, dass er einsieht, dass der Transfer auf die Literatur mit dem Opfer allein nur schwer funktioniert. Zudem fehlen ihm verständlicherweise noch die literaturwissenschaftlichen Kategorien, um solche Reflexe des religiösen Hintergrunds adäquat auszudrücken. In beiden Artikeln benutzt er die Opferthematik meist nur als Metapher und nicht im Sinne einer kreativen mythisch-rituellen Poetik.

Mit Sicherheit geht es Burkert gar nicht um eine solche Übertragung. Oft bleibt er nur im Impliziten. Damit liefert er wohl, ohne es zu wollen, ein heuristisches Instrumentarium für die Interpretation der ihm am Herzen liegenden griechischen Literatur.

\section{Burkerts Erkenntnisse als Ausgangspunkt für die Analyse einer mythisch-rituellen Poetik in der griechischen Literatur}

Burkert gibt entscheidende Anstöße, griechische Literatur auf der Grundlage eines mythisch-rituellen Substrats ganz anders zu lesen. Für diese Charakteristik der Texte prägte ich mit anderen den Begriff einer mythisch-rituellen Poetik. ${ }^{25}$ Entscheidend ist, dass griechische Autoren in Variation und Kombination einzelne Elemente von Ritualsequenzen und mythischen Erzählungen für ihre poietische Komposition zusammensetzen können. In einer solchen spezifischen Ästhetik entsteht ein freies Spiel von interrituellen und intertextuellen Bezügen. ${ }^{26}$ Vor allem wird das per-

25 Vgl. Yatromanolakis/Roilos 2003; Yatromanolakis/Roilos 2004; Bierl 2007a; Bierl/Lämmle/Wesselmann 2007a; Bierl/Lämmle/Wesselmann 2007b; zur Germanistik vgl. Braungart 1996; Braungart 2007.

26 Bierl 2001; Bierl 2007a; Bierl 2007b; Auffarth 2007; Bierl 2009a. 
formative Energiepotential, das diesen Elementen eignet, für den Aufbau effektiver und mitreißender Handlung eingesetzt.

In einem solchen Konzept ist es, wie gesehen, essentiell, dass man nicht, wie dies die Cambridge Ritualists taten, nur ein einziges pattern anlegt. Burkert ist diesbezüglich Erbe dieser Schule, indem er in Homo Necans selbst nur ein solches Muster konstruiert, das er in altsteinzeitlichen Uranfängen verortet. Und vielleicht vermeidet er auch wegen der diesbezüglichen Kritik an der myth-and-ritual-school eine konsequente Anwendung auf die Literatur. Eine solche literarische Applikation geht einher mit einer in der Religionswissenschaft sich verbreitenden Tendenz, eine polyparadigmatische Interpretation an bestimmte Feste und Riten anzulegen. ${ }^{27}$ Auch hier sind Burkerts frühe Aufsätze und Homo Necans bereits trotz des einen synthetischen pattern richtungsweisend. Zudem sind Burkerts Einzelbeobachtungen, die er zum Teil aus der Literatur als Quelle ableitet, so reichhaltig, dass diese umgekehrt auch im Sinne einer mythisch-rituellen Poetik fruchtbar gemacht werden können.

In der Folge kann ich nur einige Beispiele geben, wie in den letzten fast vier Jahrzehnten nach Homo Necans solche Übertragung geschah. Gerade auch aus Burkerts Schülerkreis sind in diesem Zusammenhang Eveline Krummen, Laura Gemelli Marciano und Christoph Riedweg zu nennen.

\section{V.1 Homerisches Epos}

Christoph Auffarth (1991) wendet das Schema des Jahresfests kombiniert mit dem Königsritual, der alle neunzehn Jahre rituell begangenen temporären Absetzung und Wiedereinsetzung des Herrschers, auf der Grundlage altorientalischer Vorbilder auf Homers Odyssee an und zeigt, wie der $\mathrm{Zu}$ stand der Marginalität als verkehrte Welt dem experimentellen Durchspielen alternativer Möglichkeiten dienen kann. Gleichzeitig sieht er bei Telemach und Odysseus das Initiationsschema in Form einer Reaktualisierung ihres Übergangsstatus am Werk. Dabei identifiziert er die Anthesterien als Rahmenfest und geht von einer partiellen Überlagerung von Odysseus mit Dionysos aus. Apollon spielt als Gott der Initiation in seinem Jahresfest für die Wiedereingliederung in Ithaka eine entscheidende Rolle. ${ }^{28}$

27 Vgl. Versnel 1993.

28 Zu Homer vgl. u. a. Forscher wie Papadopoulou-Belmehdi 1994; Cook 1995. 


\section{V.2 Lyrik und Philosophie}

Eveline Krummen oder unter anderen schon früher Greg Nagy übertrugen den Ansatz im Bereich der Lyrik auf Pindar. ${ }^{29}$ Im Falle der Vorsokratik bezieht man sich bei der Suche nach ó $\rho \chi \alpha i ́$ und $\alpha i \tau i ́ \alpha$, wie auf der Grundlage von Burkert (1969) Peter Kingsley und die neuere Forschung zeigen, auf die Denk- und Verstehensfunktion des vorliegenden Mythos sowie zum Teil auf in der Orphik vorhandene mythisch-religiöse Ausdrucksformen und Wege der Reflexion. ${ }^{30}$ Mysterienkulte bestimmen vor allem die Denk- und Darstellungsweise von Parmenides und Empedokles. ${ }^{31}$ Desgleichen ist Heraklit von orphischen Einweihungsvorstellungen nicht unberührt. ${ }^{32}$ Platon setzt bekanntlich an zahlreichen Stellen Mythen ein und schafft zur Verständigung in dichterischem Verfahren neue Mythen. ${ }^{33}$ In einigen Dialogen, vor allem im Symposion, Phaidros und Phaidon, kann man nachweisen, wie die Mysterienterminologie die narrative Struktur prägt. ${ }^{34}$

\section{V.3 Geschichtsschreibung}

Im Bereich der frühen griechischen Geschichtserzählung werden neuerdings die Analogien von Mythos, Ritual und Geschichte deutlicher erkannt. In der Suche nach Ursachen und Anfängen tritt, wo man Leerstellen zu füllen hat, die mythische Fiktion ein, die sich schließlich von Aitiologien löst und produktiv Erzählung fortspinnt. In freier Anverwandlung werden solche Elemente immer neu variiert, so dass mythisch-rituelle Bausteine auch die Historiografie bestimmen. Mythos und Historie greifen

29 Vgl. u. a. Nagy 1990, bes. 116-135; Krummen 1990; zu Sappho vgl. u. a. Bierl 2003; Yatromanolakis 2003; Yatromanolakis 2007; Nagy 2007.

30 Vgl. u. a. Kingsley 1995; Riedweg 1995; Cerri 1999; Morgan 2000, bes. 46-88; Kingsley 2000; Kingsley 2003; Bierl (in Vorbereitung); Gemelli Marciano 2007; Gemelli Marciano 2008.

31 Zu Empedokles vgl. u. a. Riedweg 1995; Kingsley 1995; Bierl (in Vorbereitung); zu Parmenides vgl. nach Burkert 1969 u. a. Kingsley 2000; Kingsley 2003; Gemelli Marciano 2008.

32 Vgl. Seaford 1986, 14-20; Schefer 2000 (mit älterer Literatur 46 Anm. 1), die Heraklits Fragmente allerdings in überzogener Weise als "echten Mysterienlogos" (73) deutet. Vgl. nun Gemelli Marciano 2009.

33 Vgl. u. a. Murray 1999; Janka/Schäfer 2002.

34 Vgl. Riedweg 1987, 1-69, der für einen metaphorischen Bezug plädiert. Zur strukturellen Verwendung von athenischem Fest- und Kultmaterial bei Platon vgl. auch Krummen 2002; Krummen 2007. 
symbolisch auf ein ähnliches kulturell-symbolisches Ausdrucksreservoir zurück und zielen auf den nämlichen Effekt. ${ }^{35}$ Gerade bei Herodot erkennt man zunehmend, dass er in besonderer Weise mit mythisch-rituellen Erzählmustern operiert. Das Burkertsche Atreusmahl im Kontext des Werwolfkomplexes wird beispielsweise im Fall des Harpagos (1.108-119) eingesetzt, um den Übergang der Dynastien zu markieren. Überhaupt scheint Herodot mit Vorliebe Geschichten des rite de passage als erzähltechnische Untermalung für Umbrüche und Wandel einzusetzen. Adrastos (1.24-45) erlebt beispielsweise in eigenartiger Montage eine Art 'Knabentragödie'. Und die Geschichte des jungen Kyros (1.107-130) und die Aussetzungsgeschichte des Kypselos (5.92) dienen in bewusster Aufnahme von mythischen und rituellen Folien und Strukturen erneut der narrativen Betonung entscheidender Schlüsselstellen. Episoden des Wahnsinns und eine diesbezüglich spezifisch dionysische Zeichnung, die durch das Zusammenfallen und die wechselseitige Verschränkung von Polaritäten gekennzeichnet ist, finden sich bei Kambyses im dritten Buch und bei dem Spartanerkönig Kleomenes (6.75-84). Orphisch-Dionysisches sowie Initiationsmuster kann man im grausamen Ende von Anacharsis und Skyles erkennen (4.76-80). An zahlreichen Stellen wird deutlich, dass Herodot selten nur mit einem pattern operiert, sondern in polyparadigmatischer Weise Initiation, rite de passage, Neujahr, kosmisches Jahresfest, Königsritual, Opfer und Mysterien vermengt und unter Zugabe anderer paralleler mythischer Erzähltraditionen aufbereitet. ${ }^{36}$

\section{V.4 Tragödie}

Im Bereich der Tragödie hat Burkert (1966b, 117-121; 1985a) den Schritt zur literarischen Interpretation gemacht. Sein anthropologisches Ursprungsmodell ist inzwischen auf vielerlei Art und Weise ergänzt worden. Burkert sieht das Opfermotiv in seinen Anwendungsversuchen lediglich

35 Dazu Alexiou 2002, 156-157. Allgemein dazu vgl. Calame 2003; zu Herodot vgl. u. a. Calame 2000, 145-167; Stadter 2004; Chiasson 2005; Wesselmann 2007; zu Thukydides, bei dem man dies aufgrund seiner vermeintlich objektiven Geschichtsschreibung am wenigsten erwartet hätte, vgl. schon Cornford 1907.

36 Unter anderem in Aufnahme Burkertscher Interpretationsschlüssel wird Katharina Wesselmann unter meiner Betreuung zu diesem Aspekt demnächst eine vielversprechende Dissertation einreichen, die sie dann auch bald in Buchform vorlegen wird. 
als Metapher und erkennt noch nicht das narrative und performative Potential für das Theaterspiel. ${ }^{37}$

Helen Foley hat fast zwanzig Jahre später diverse religionswissenschaftliche Opfer-Ansätze verbunden und die Eigengesetzlichkeit der Literatur, ihre Mittelbarkeit und ihr Abgelöstsein von der Realität, berücksichtigt. ${ }^{38}$ Schon zur gleichen Zeit wie Burkert hat Froma Zeitlin (1965; 1966) die Verwendung des Opfer-Motivs in der Tragödie als Perversion des Rituals analysiert. Ferner hat man nach Burkert vor allem den strukturbestimmenden Aspekt des Opfers in der literarischen Verarbeitung herausgearbeitet. ${ }^{39}$ Pierre Vidal-Naquet (1969) assoziiert fast zeitgleich mit Burkert (1966b; 1972, bes. 8-96) die Motive von Jagd und Opfer. Die Sündenbocktheorie in Verbindung mit dem Thargelien-Ritual des $\varphi \alpha \rho \mu \alpha-$ кós ist nach der Girardschen Analyse ein weiterer Schwerpunkt der Opferanalyse in der Tragödie. ${ }^{40}$ In der attischen Tragödie wird, wie nach Zeitlin nun beispielsweise Albert Henrichs (2000; 2004; 2006) und John Gibert (2003) zeigen, in der übersteigerten Verkehrung das Gewaltpotential des Tieropfers dramaturgisch umgesetzt. Mord und Totschlag der Figuren werden dabei mit Opferterminologie signalhaft aufgeladen und so performativ ausgespielt.

Die Tragödie soll wie die Komödie aus dem Kult des Dionysos hervorgegangen sein und besitzt auf institutioneller Ebene eine enge Verbindung mit dieser Gottheit, die, wie betont wurde, für Burkert zur heimlichen Hauptfigur avanciert. Daher hat man dann bald jenseits der leidigen Frage des Tragödienursprungs seine besondere Funktion in den tragischen Texten selbst zu untersuchen begonnen. ${ }^{41}$ Manche sehen den Zusammenhang mit der Tragödie ähnlich wie Burkert in einer allgemeinen anthropologischen Beziehung, gerade auch in der Auflösung und Wiederherstellung der Ordnung. ${ }^{42}$ In Verbindung mit der modernen Literaturwissenschaft erkannte man in Dionysos auch eine metadramatische Dimension. ${ }^{43}$

37 Vgl. auch Susanne Gödde in diesem Band.

38 Vgl. die gute Einleitung von Foley 1985, bes. 17-64.

39 Zum strukturbestimmenden Aspekt vgl. u. a. Pucci 1977; Seidensticker 1979; Henrichs 2004.

40 Vgl. Vernant 1972, 114-131, bes. 114-119 (in S. OT); Segal 1982, $36-54$ (in E. Ba.).

41 Vgl. u. a. Bierl 1991; Aronen 1992.

42 U. a. Brelich 1975; Aronen 1992; Bierl 1991; des Bouvrie 1993.

43 Segal 1982, 215-271, 369-378; Bierl 1991, 111-218. 
Nach Burkert war zudem selbst eine ritualistische Lektüre der Tragödie in den Spuren der Cambridge Ritualists wieder möglich. Richard Seaford ist einer der wichtigsten Vertreter einer solchen Richtung. Er postuliert für die Tragödie im Vorkommen des Dionysos und des Dionysischen ein einheitliches Handlungsmuster politisch-gesellschaftlicher Provenienz, das die Zerstörung des königlichen Haushalts und die nachfolgende Herausbildung einer kollektivistischen Polisordnung zum Inhalt hat. ${ }^{44}$ Zudem zieht er besonders die Mysterien als Handlungssubstrat der Tragödie heran. Die Euripideischen Bakchen werden in solchen Konstrukten, wie es unbemerkt bei Burkert geschah, zum Schlüsseltext, aus dem man in einem Zirkelschluss die Theorie ableitet. Seaford deutet dementsprechend die Bakchen, die angeblich den Gattungsursprung aus bakchischen Einweihungsriten reflektieren, als Dramatisierung des Aition von thebanischen Dionysosweihen. Neben den in Pubertätsweihen wurzelnden Mysterien fließen laut Seaford in die Komposition der Bakchen unter anderem Jagdund Opferbräuche, Klage-, Trauer- und Hochzeitsriten, das Pharmakosritual und Vegetationsbräuche ein. ${ }^{45}$

Auf andere Riten neben dem für die Tragödie konstitutiven Opfer geht Burkert kaum ein, bespricht diese allgemeinen Riten freilich in seiner Griechischen Religion (Burkert 1977) auf dem neuesten Stand der Religionswissenschaft. Zunehmend erkennt man, dass die Tragödiendichter unter anderem Hochzeitsriten und ihre Verbindung mit Todeserfahrungen, ferner Hikesie- und Supplikationsszenen, Reinigungen, Segnungen, Beschwörungen, Verfluchungen, Jagdriten, Heilungen, Gebet und Gebetsreihen, Eide, magische Binderituale, Bestattungsbräuche, Klagen und Threnoi, Tanz, Agone und Prozessionen für ihre Texte transformieren. ${ }^{46}$ Das tragische Spiel, das selbst im rituellen Kontext aufgeführt wird, integriert diese Riten meist in Chorpartien als direkt am Körper ausagierte lebensweltliche Handlung, wobei performativ gewissermaßen 'Ritual im Ritual' entsteht. ${ }^{47}$

44 Seaford 1993; Seaford 1994; Seaford 1996.

45 Seaford 1981; Seaford 1994; Seaford 1996.

46 Vgl. den Überblick mit Belegen bei Bierl 2007a, 30-33. Zu Prozessionen vgl. Bierl 2010a.

47 Vgl. u. a. die vorbildlichen Studien von Wolff 1992 und Krummen 1998. Einzelstudien, wie die von mir betreute Dissertation von Valentina Luppi zu Euripides' Helena werden in der Zukunft das Zusammenspiel von einzelnen Riten und Paradigmen noch deutlicher aufweisen. 


\section{V.5 Komödie - der neue Paradefall}

Aufgrund der Konzentration auf das Ernste und Erhabene verzichtet Burkert ganz darauf, seine Gedanken auf die Komödie oder das Satyrspiel anzuwenden. ${ }^{48}$ Freilich wirkt er durch die zahlreichen Verweise auf die Alte Komödie, die häufig als religionswissenschaftlicher Quellenbeleg in Homo Necans zitiert wird, erneut als Inspirationsquelle für spätere Arbeiten. Angus Bowie (1993), Christoph Auffarth (1994; 1999; 2004; 2007) und ich selbst (Bierl 1994; 2001; 2004) versuchten folglich Aristophanes als eine Art Musterfall einer mythisch-rituellen Poetik einzuführen.

Gerade bei der Beschreibung des Opferverlaufs rekurriert Burkert (1972, 10-14) auf eine Szene im Aristophanischen Frieden. Dionysos als Gott des Theaters ist selbstverständlich auch in der Komödie wirksam. Das dunkle Szenario Burkerts hat selbst für diese heitere Gattung unterbewusst weitergewirkt. Xavier Riu (1999) leitet dementsprechend das Dionysische in der Komödie aus einer strukturalistisch-soziologischen Bakchen-Lektüre auf den Spuren der Pariser Schule ab, die trotz aller Gegensätze zu Burkert sich mit ihm in einer von Nietzsche beeinflussten lebensdramatischen Deutung trifft, und überblendet es mit dem alten Schema Cornfords (1914). ${ }^{49}$ In der Aufnahme des Dionysos als Prinzip des Anderen in die Polis wird nach Riu die Auflösung der Ordnung ganz im Sinne Burkerts durchgespielt. Das Dionysische ist allerdings nicht nur Chiffre für Gewalt, Ekstase, Sparagmos, Opfer und Brutalität, sondern beinhaltet auch positive Werte, insbesondere die Einheit der Polis in Gemeinschaft stiftender Festfreude. ${ }^{50}$ Gerade im Satyrspiel und in der Komödie ist Dionysos als Zeichen des liminalen Anderen zudem Ausdrucksmittel der ebenso das dionysische Rahmenritual beherrschenden Atmosphäre von heiterer Ausgelassenheit in Tanz und Spiel sowie von berauschter Stimmung bei Wein.

Das für Burkert so zentrale Opfer und Störungen im kanonischen Ablauf des Rituals bestimmen durchaus auch die Struktur der Alten Komödie. Ich denke dabei beispielsweise an die Acharner, den Frieden und

48 Zum Satyrspiel vgl. Seaford 1984; Bierl 2006; Lämmle 2007 und die Dissertation mit dem Titel "Poetik des Satyrspiels", die Rebecca Lämmle unter meiner Betreuung in Basel abgeschlossen hat und demnächst in Buchform erscheint.

49 Zu Dionysos in den Fröschen vgl. Bierl 1991, 27-44; Lada-Richards 1999.

50 Vgl. Bierl 1991, bes. 18-20, 45-110, bes. 49-54; Isler-Kerényi 2001. 
die Vögel des Aristophanes. ${ }^{51}$ Durch verkehrte Gründungsopfer kann die Inversion der Ordnung ausgedrückt werden. Umgekehrt kann der Vollzug eines Opfers gegen Ende einer Komödie als Zeichen der Rückkehr zur Ordnung gelesen werden. ${ }^{52}$ Neuerdings wendet man sich zunehmend von den vorherrschenden dunklen Szenarien der Gewalt, Angst und Schuld ab, die Burkerts Opferanalyse bestimmen, und bewertet auch auf der Grundlage ikonografischer Darstellungen das Opfer im Festkontext positiver. Dabei geraten die ausgelassene Freude am Fleischkonsum, das Lachen und die komischen Elemente in den Blick, die häufig auf Vasenbildern zum Ausdruck kommen. ${ }^{53}$ Dies hat vor allem Auswirkungen auf die Interpretation der Komödie, die sich insbesondere durch diese heiteren Aspekte des Dionysosfestes auszeichnet.

Die Alte Komödie ist freilich nicht nur von Heiterkeit bestimmt, sondern das in Homo Necans entwickelte Modell der dionysisch gefärbten Auflösung und Wiederherstellung der Ordnung erweist sich auch für diese Gattung als ein zentrales Konzept. Die grundsätzliche Andersartigkeit der dort inszenierten Gegenwelten ist nicht notwendigerweise auf das dionysische Rahmenritual zurückzuführen, sondern lässt sich allgemeiner mit der Festlichkeit, dem Spiel, der verkehrten Welt und mit Ausnahmeritualen der Lizenz erklären. ${ }^{54}$ Im Hintergrund stehen ethnologische Vorstellungen von brauchtümlichen Festen und theatralen Formen, in denen die Gesellschaft kurzfristig die etablierte Ordnung verlässt und in Phantasien eine groteske Anderwelt im Rückfall auf Atavismen experimentell durchspielt, um dadurch im Kontrast die bestehenden Werte und Normen letztendlich zu bestätigen. ${ }^{55}$ Mittels eines Sturzes in primordiale Kulturstufen und urtümliche Vorstellungen wird ein nicht auf das Reale bezogenes groteskes Leben der Dystopie und Utopie im Funktionellen inszeniert. ${ }^{56}$ Solche karnevalesken Formen der verkehrten Welt kann man in der Antike am besten vom pragmatischen Bezug auf bestimmte Ausnahmefeste ableiten. Die Kennzeichen der temporären Auflösung der Ordnung (u. a. Tod, Obszönität, Wildheit, tierisches Verhalten, Tanz, Utopie, Alt-Neu, Absetzung der Götter, Sklavenfreiheit, Gewalt) lassen sich mit

51 Vgl. auch Sfyroeras 2004.

52 Vgl. u. a. Bowie 1993, Index s. v.; Riu 1999, Index s. v.

53 Vgl. u. a. Peirce 1993.

54 Hoffman 1989; Farioli 2001.

55 Vgl. Bierl 2002a.

56 Vgl. Bierl 2002a; Bierl 2004 mit Verweis auf Münz 1998, bes. 99-101, 282. 
den drei großen religionswissenschaftlichen Paradigmen (Initiation, Neujahr und Fruchtbarkeit) erklären. Der dionysische Zusammenhang wurde zum Teil heortologisch durch eine referentielle Verbindung mit den in vielen Details exzeptionellen Anthesterien gesucht. ${ }^{57}$ Derartige Bezüge können selbstverständlich von der Rahmung her auch den Inhalt des Spiels als 'Mythos' beeinflussen. Die Anthesterien, die in Burkerts Homo Necans (1972, 236-269) einen so herausgehobenen Platz haben, wirken dann in zahlreichen Einzelbezügen auf den Plot zurück. Der Rückfall in die phantastische Anderwelt wird dann als Rückkehr der Anderen, der Toten und Barbaren, der grotesken Masken und der schrecklichen Weiber ausgespielt. Bowie (1993), Auffarth (1994; 1999; 2004; 2007) und ich selbst (Bierl 1994; 2001) gehen auf die mythisch-rituelle Durchdringung ein, wobei Burkert immer wieder Anstöße gibt. Bowie und ich greifen dabei vor allem wiederholt auf das in Aufnahme von Jane Harrison und Angelo Brelich von Burkert weit vertretene Paradigma der Initiation zurück. In der Literatur wird dieser Motivkomplex häufig durch die Reaktualisierung und die Umkehrung des Initiationsablaufs verarbeitet. ${ }^{58}$ Am Beispiel der Thesmophoriazusen habe ich selbst (Bierl 2001, 105-299) ausführlich gezeigt, wie die Frauen am Thesmophorienfest konzeptionell den Status der jungen Mädchen an der Schwelle zur Frau wiederholen und Aristophanes auf diesem Schema sein komplexes Stück aufbaut. Burkerts Aufsatz (1970) über das Jahresfest auf Lemnos und seine entsprechenden Ausführungen in Homo Necans (1972, 212-218) haben zudem die Deutung der Lysistrate und der Ritter des Aristophanes inspiriert;59 die Ekklesiazusen werden ferner auf Anregung Burkerts $(1972,164)$ in Verbindung mit den Skiren und Panathenäen gesehen, welche den Festzyklus des attischen Jahresübergangs mitbestimmen. ${ }^{60}$

Anhand des Konzepts einer mythisch-rituellen Poetik erkennt man, dass Komödienautoren ganze Abläufe mitsamt Einzelritualen zur spielerischen Konstruktion von Handlungsstrukturen verwenden, wie beispielsweise den Festrhythmus von den Skiren bis zu den Panathenäen, der von Burkert (1972, 153-177) so deutlich als Neujahrskontext herausgearbeitet

57 Vgl. u. a. Auffarth 1994; Bierl 1994.

58 Zur Reaktualisierung vgl. Bierl 2001, u. a. 267, 276-287, 313, 318 Anm. 48, 341 Anm. 105; zur Umkehrung vgl. Bowie 1993, 78-101, 102-112.

59 Zur Lysistrate vgl. Martin 1987; Bowie 1993, 178-204; zu den Rittern vgl. Bowie 1993, 66-74.

60 Vgl. Bowie 1993, 254-267. Vgl. auch Auffarth 2004. 
wurde. ${ }^{61}$ Gerade das dort vorkommende Arrhephoren-Ritual sowie andere repräsentative weibliche Initiationskulte spielen für die Interpretation der Lysistrate eine wichtige Rolle, in der sich erneut die älteren Frauen in den Status der Mädchen vor der Hochzeit zurückversetzen. Burkerts Aufsatz Kekropidensage und Arrhephoria (1966a) erweist sich mit dem geschilderten Szenario am Nordhang der Akropolis als wahre Fundgrube für eine mythisch-rituelle Deutung der Lysistrate.

Für die attische Komödie sind besonders Kulte der eponymen Polisgöttin Athene von Bedeutung, ebenso Kulte der Artemis und des Dionysos. Erwartungsgemäß sind Elemente der dionysischen Feste, wie zum Beispiel der Anthesterien, Lenäen, der Großen und der Ländlichen Dionysien, die in die Handlung projiziert werden, häufig spielbestimmend. Spezifische Jahres-, Einweihungs- und Fruchtbarkeitsfeste können ebenso für die Plotgestaltung herangezogen werden. Hier ist besonders an die Mysterienfeiern in Eleusis, die Thesmophorien, das Adonisfest oder die Panathenäen zu denken. Zudem können strukturelle Zusammenhänge mit Mysterienkulten, insbesondere mit Eleusis und orphisch-bakchischen Einweihungskulten, um deren Erforschung sich Burkert so verdient gemacht hat, in das Gewebe der Komödie einfließen, wie es z. B. im Fall der Frösche geschieht. ${ }^{62}$ Selbst in der politischen Komödie kann der Mythos beispielsweise die Sage der Sukzession der Göttergeschlechter, der Gigantomachie oder der Herrschaft der Amazonen - eine konstitutive Funktion erhalten, wodurch der Rückfall in die Anderwelt unterstrichen wird. ${ }^{63}$ Für die Vögel spielt natürlich der Mythos von Tereus-Epops mitsamt seinem von Burkert (1972, 201-207) herausgehobenen Gewaltpotential eine große Rolle.

Das Fruchtbarkeitsparadigma ist seit den 1960er Jahren vor allem unter dem einflussreichen Diktum Burkerts fast ganz außer Mode gekommen, obwohl der bäuerliche Hintergrund als ökonomische Basis gerade in der archaischen und klassischen Zeit die Lebenswelt Athens weitgehend bestimmt. Doch verspricht eine Rückkehr zu Wilhelm Mannhardt, James Frazer oder Francis Cornford für die Interpretation der Alten Komödie einige Ergebnisse, ${ }^{64}$ selbstverständlich nur auf dem Niveau heutiger

61 Zur Lysistrate vgl. Bierl 2007d.

62 Vgl. Lada-Richards 1999, bes. 45-122; Riu 1999, bes. 92-94, 102-103, 136-139, 141.

63 Vgl. Bowie 1993; bes. zu den Vögeln vgl. Hofmann 1976; Zannini Quirini 1987.

64 Vgl. Bierl 2007a, 29-30. 
Methoden. Aristophanes bietet sich erneut für die Anwendung des agrikulturellen Interpretationsmodells besonders an - auch in Verbindung mit den Mysterien von Eleusis -, zumal viele seiner Komödien voll von Fruchtbarkeitsmotiven sind. Ich denke vor allem an die Acharner, den Frieden, die Frösche und den Plutos. In diesem Zusammenhang ist das Anodos-Schema hervorzuheben, also die Hervorholung einer unterirdischen Göttin als symbolischer Ausdruck einer nahezu utopischen Rückkehr zur Fruchtbarkeit, zu Reichtum und Gesundheit. ${ }^{65}$

Die von Karl Meuli, Eric Dodds und Burkert angestoßenen Überlegungen zum griechischen Schamanismus sind in der Religionswissenschaft und in der Verbindung von Literatur und Religion wichtig geworden, wobei man bisher den Begriff meist auf charismatische Weise und auf Vorsokratiker anwandte. ${ }^{66}$ Trotz skeptischer Stimmen ${ }^{67}$ ließ sich Burkert hier kaum beirren und betonte weiterhin die Gültigkeit des anthropologischen Konzepts, ganz gleich, ob es wirklich einen institutionellpragmatischen Bezug zu solchen Figuren gab oder nicht. Richtigerweise bezeichnet er den griechischen Schamanen mit dem griechischen Terminus róns (Burkert 1962b). Die Forschungen, die auch nach Burkert 'schamanistisches' Gedankengut in der archaischen Dichtung aufdecken, zielen weiterhin eher auf die Erklärung des Ursprungs als darauf, die besonderen Strukturprinzipien und die generische Funktion von Texten damit zu verdeutlichen. Bowie (1993, 112-124) deutet auf Anregung Burkerts die Gestalt des vorsokratisch-sophistischen Weisen Sokrates in Aristophanes' Wolken als yóns. ${ }^{68}$ Ich selbst (Bierl 2007c) ging hingegen der Frage nach, wie sich anhand 'schamanistisch' gezeichneter Figuren im attischen Theater ein Spiel mit dem Anderen und dem Selbst einstellen kann. Damit verstehe ich 'Schamanismus' in der Komödie als theatrales Gedankenexperiment. Die Alte Komödie greift, so meine These, im komischen Rückfall in ein vorzivilisatorisches Stadium auf dieses uralte

65 Zum Motiv: in den Thesmophoriazusen vgl. Bierl 2001, 139 mit Anm. 85; in den Fröschen vgl. Lada-Richards 1999, 81-84, 106-108, 114; im Frieden vgl. Bowie 1993, 143-146.

66 Vgl. Meuli 1935; Dodds 1951, 135-178; Burkert 1962a, 98-142, 324-325 (zu frühen Wanderpriestern, Sehern, Dichtern und Philosophen wie z. B. Pythagoras, Abaris, Aristeas, Epimenides, Orpheus, Parmenides, Empedokles); West 1983, 3-7, 49, 143-150 (bes. Orpheus); Kingsley 1995 (zu Empedokles).

67 Vgl. u. a. Bremmer 1983, 25-48, 52; Graf 1987; Zhmud 1997, 107-116.

68 Vgl. auch Guidorizzi 1996, 220, 224, 229 und zum 'Schamanismus' der initiatorischen Riten im Umfeld der Denkerstube des Sokrates 199, 206, 222, 229, 235. 
Konzept zurück, das gleichzeitig als Substrat in der Volkskultur und im einfachen Brauchtum weiterlebt. Die 'Reminiszenz' solcher atavistischer Verhältnisse auf der komischen Bühne stellt demnach ein artifizielles poetisches Konstrukt dar, womit das Barbarische der magoi in die Polis hereingeholt wird, um ihr komplementäre Welten zu eröffnen. Im Vergleich zum Schamanismus stößt die von nordamerikanischen Mythen stammende Vorstellung des Tricksters in der Gräzistik auf relativ große Akzeptanz. Neben Versuchen Burkerts (1984c, 840-845), der das Konzept auf Hermes und Prometheus in der literarischen Darstellung anwandte, findet es im Bereich der Komödien-Forschung breite Zustimmung. Wie Hermes, der beispielsweise im Frieden und im Plutos dementsprechend agiert, parallelisiert man insgesamt den komischen Helden in seiner grotesken Körperlichkeit gerne mit dem Trickster. ${ }^{69}$

\section{V.6 Roman}

Burkert ist wohl immer noch von der Mysteriendeutung seines Mentors Reinhold Merkelbach überzeugt. ${ }^{70}$ Einen eigenen Akzent für diese Gattung kann er setzen, indem er Apuleius' Psyche in der berühmten Geschichte von Amor und Psyche ausführlich mit seinem Konzept der "Mädchentragödie" in Beziehung rückt. ${ }^{71}$ In eigenen Beiträgen (Bierl 2007b; Bierl 2010b) gehe ich einem bio-rituellen Ansatz der Gattung auf der Grundlage einer initiatorischen Deutung mit literaturwissenschaftlichen Methoden nach. Auch der antike Roman kann deutlich in einer mythischrituellen Poetik interpretiert werden, wobei besonders die Initiation in Form der Pubertätskrise der beiden blutjungen Helden in den Blick genommen wird. Initiatorische Riten und der Roman, der wiederum auf mündlich überlieferten traditionellen Wundererzählungen basiert, thematisieren und umspielen den zentral empfundenen Lebenseinschnitt der Hochzeit und die Krise der erwachenden Sexualität. Burkert hat diese Thematik auf eindringliche Weise in seinem Aufsatz Kekropidensage und Arrhephoria (1966a) gezeigt. Diese Zusammenhänge werden auch im Roman häufig aus der Perspektive des jungen Mädchens beschrieben. Romane, volkstümliche Geschichten und entsprechende Riten verarbeiten diese Themen in traumartigen Sequenzen in positiver und negativer

69 Vgl. u. a. Brelich 1975, 114-117; Zannini Quirini 1987, 19; Riu 1999, 3, 244-245.

70 Vgl. Burkert 1987b, 66-67.

71 Vgl. Burkert 1996a, 69-79. 
Weise. Ängste, Alpträume von monströsen Erscheinungen und Szenarien von Blut und Opfer sind mit euphorischen Phantasien vermengt. Liebe ist insgesamt für die Gattung des Liebesromans konstitutiv. Von Beginn an finden sich die beiden Helden in einer Schlaufe destabilisierender Gedanken und in marginalen Räumen, am Ende steht das Ziel der Hochzeit. ${ }^{72}$ Hinter den Romanhandlungen und hinter den volkstümlichen Geschichten steht meiner Meinung nach eine Art "biologische Spur", eine psychoanthropologische Grundlage. Diese bildet also wirklich eine "Mädchentragödie", freilich weniger im Sinne eines biologisch geprägten Handlungsprogramms, wofür Burkert (1996a, 69-79) plädiert, als vielmehr im Sinne eines losen Clusters von Erzählmotiven, die in freier Variation und Kombination zusammengesetzt werden können. ${ }^{73}$

Wie beispielsweise Achilleus Tatios schon über den Namen seiner Heldin Leukippe das unsagbare Geschehen einer Mädchentragödie sowie die Auflösung der Ordnung als Schreckensphantasie inszenierte, fast als hätte er die tragisch-dionysische Interpretation Burkerts bereits vor sich liegen, habe ich an anderer Stelle dargestellt. ${ }^{74}$ Es ist ein schönes Beispiel dafür, wie der Roman das Szenario von Mythen und damit verbundener Riten für seine Plotgestaltung produktiv verwendet. In einer faszinierenden Passage malt Burkert in Homo Necans $(1972,189-197)$ die Geschichte der Minyastochter Leukippe in Orchomenos aus, die sich zusammen mit ihren Schwestern Arsippe und Alkathoe zunächst Dionysos widersetzt. Aufgrund der Rache des Gottes zerreißen sie in einer an die Bakchen erinnernden Konstellation gemeinsam Leukippes Sohn Hippasos - schon über den Namen erkennt man den Reflex eines Pferdeopfers. Plutarch (Quaest. Graec. = mor. 299e-f) assoziiert den Mythos mit einem Verfolgungsritual, wobei die 'Schwarzen' ( $\Psi$ o $\lambda$ ó $1 \varsigma$ ), welche die trauernden Gatten der Minyaden verkörpern, die 'Mörderinnen' ('O $\lambda \varepsilon \hat{\imath} \alpha \iota$ ) verfolgen. Zuletzt werden die Frauen in Nachtvögel verwandelt. Manches erinnert an die grausamen Kämpfe zwischen den ägyptischen Räuberbanden um das Mädchen bei Achilleus Tatios. Die Minyaden werden auch bei Burkert mit der weiteren Mädchentragödie der Proitiden parallelisiert. Lysippe entspricht in diesem Falle Leukippe: Das 'entfesselte Pferd' tritt mit Iphinoe

72 Vgl. Bierl 2007b, bes. 265-276. Zu Apuleius vgl. Bierl 2009b; Bierl 2010b. Zu einer Biopoetik der Mädchentragödie vgl. auch Michael Neumann in diesem Band.

73 Zu Longos vgl. nun auch Bierl 2009a.

74 Vgl. dazu schon Bierl 2007b, 267-269. 
und Iphianassa auf, mit Mädchen, die an der Grenze zum Frausein mit männlicher Kraft handeln. Unmittelbar vor ihrer Hochzeit werden sie von Hera verfolgt. In totaler Auflösung der Ordnung treiben sie Unzucht in allen erdenklichen Formen. Sie rasen im Wahn nackt durch die Peloponnes, und halten sich in ihrer Geilheit für Kühe. Hera bedeckt ihre Gesichter mit einer weißen Substanz und sie bekommen einen hässlichen Ausschlag. Zudem lässt sie ihnen das Haar ausfallen. Die schrecklichen Weibergruppen bestreuen sich im Ritual oft mit Mehl. Manches erinnert an groteske Masken alter, die Herrschaft kurzzeitig an sich reißender Vetteln im Umfeld der Artemis Orthia. Nach einer anderen Version versagen die Proitiden Dionysos die Ehre. Der ebenfalls dionysische Priester Melampus, der 'Schwarzfuß', verfolgt in ekstatischen Tänzen die Rasenden zusammen mit den Epheben der Polis und heilt schließlich die wild gewordenen Frauen. Dafür erhält er ein Proitos-Mädchen zur Frau und wird König. Bei der ungestümen Verfolgung kommt allerdings Iphinoe ums Leben, der zu Ehren die Agrionia eingerichtet werden. Allein über den Namen Leukippe wird also das ganze Spektrum der Initiationsphantasie aufgerufen. Zentral ist die polare Spannung zwischen dem keuschen und dem sexuell-entfesselten Mädchen, das die Ehegöttin Hera beleidigt. Dionysos als alternativer Auslöser der Entwicklungen ist ebenso im Roman des Achilleus Tatios verankert. Die Pole zwischen dem reinen Weiß und dem befleckten Schwarz schlagen ineinander um und sind miteinander verschränkt. Der Heiler Melampus, der 'Schwarzfuß' im Bereich des 'Anderen', ist sowohl gut als auch schlecht. In tänzerischer Verfolgung versucht er das Mädchen als wildes Fohlen unter das Joch der Ehe zu führen. Damit bringt er den aufgelösten Zustand in die rechte Ordnung. Doch als jagender Mann bedroht er zugleich die Keuschheit des Mädchens. Dies entspricht exakt der Rolle der Männer im Roman. Selbst Kleitophon trachtet zunächst im Roman des Achilleus Tatios ständig danach, sein geliebtes Mädchen sexuell zu bedrohen oder mit anderen Frauen Liebesabenteuer einzugehen, bis es zuletzt zum Happy-End einer Hochzeit mit Leukippe kommt. Kleitophon stellt zudem die weitere Gefährdung ihrer intakten Jungfräulichkeit dar. Das 'Selbst' und das 'Andere', der griechische Held Kleitophon und die männlichen Dritten, fallen damit partiell zusammen. Die sexuelle Aggressivität des Mannes, die nach der Logik des Liebesromans immer wieder auf andere Figuren verschoben wird, stellt die Gewalt dar, die zur Liebe charakteristischerweise gehört. Doch wie der Heiler Melampus kann der 'wilde Mann' Leu- 
kippe von der Mania mit einem Gegengift lösen, in Gestalt des zuletzt in sie verliebten Sklaven des ägyptischen Magiers Gorgias. Leukippe war nämlich durch die ungemischte Dosis des Liebestranks, den Gorgias' Diener ihr für seinen Herrn eigentlich in gemischter Form mittels des Weingottes Dionysos hätte verabreichen sollen, wie ihr mythisches Modell wahnsinnig und lasziv geworden. In diesem Zustand hat die Heldin sogar ihre Scham entblößt (4.9.2). Gleichzeitig ist sie jedoch eigentlich der Inbegriff der Keuschheit. Diese Haltung nimmt sie darauf in Ägypten an, was durch die Signifikanten des Weißen und Kahlen symbolisiert wird. Sie wird Opfer der Gewalt und Sexualität, die sie zum Teil selbst verkörpert. Das unsagbare Opfer des Mädchens wird durch mehrere grausame Scheintode in Szene gesetzt. Es wird also an diesem Beispiel deutlich, wie im Liebesroman widersprüchlich-paradoxe Signifikantenketten generiert werden, die das Thema der aufkommenden Sexualität und der einschneidenden Krise der Hochzeit aufschieben, umspielen, durcharbeiten sowie die gesellschaftlichen Normen gleichzeitig in Frage stellen und bestätigen. In einer relativ späten Literaturgattung wird eine mythisch-rituelle Poetik in besonderer Weise wirksam, wobei der Komplex der Burkertschen Mädchentragödie sowie nahezu tragisch-dionysisch gezeichnete Szenarien aus dem dritten, mit "Auflösung und Neujahrsfest" betitelten Kapitel von Homo Necans in der spezifischen Verknüpfung mit dem weiblichen Initiationsparadigma für eine literarische Interpretation fruchtbar gemacht werden können.

\section{Würdigung, Geburtstagskolloquia und Zusammenfassung}

Walter Burkert hat sich als Klassischer Philologe auf dem Gebiet der exemplarischen griechischen Religionswissenschaft vielleicht zum bedeutendsten noch lebenden Geisteswissenschaftler der letzten zwei Generationen entwickelt, vor allem auch deshalb, weil er die Brücke zur Naturwissenschaft schlägt. Wie nur ganz wenige hat er den Dialog zwischen diesen Forscherwelten beflügelt. Vor allem hat er eine ungeheure Wirkung in weiten gebildeten Kreisen erzielt, da seine Studien den Menschen in seinem Fundament zu erklären versuchen. Immer wieder kreisen Burkerts Forschungen um die Fragen der Humanität schlechthin, um die Erklärung und Bewältigung von Gewalt und Krieg. Kaum ein anderer Kultur- und Geisteswissenschaftler hat es zu solchen Auszeichnungen und zu so gro- 
Ber öffentlicher Anerkennung gebracht. Überall wird er zitiert, seine Erkenntnisse gehen ein in Theaterprogramme, in künstlerische Produktionen und Performances.

Im Blick auf die ihm gewidmete Festschrift zum fünfundsechzigsten Geburtstag mit dem Titel Ansichten griechischer Rituale (Graf 1998) wird das faszinierende Spektrum seiner Wirkung deutlich. Rituale und die Verbindung zur Tragödie und Philosophie stehen dabei zu Recht im Mittelpunkt. Burkerts Schüler und enge Weggefährten reflektieren hier seine wissenschaftlichen Errungenschaften.

Die Acta des Kolloquiums zum fünfundsiebzigsten Geburtstag am Istituto Svizzero in Rom (2006) sind eben in dem von Christoph Riedweg (2009) herausgegebenen Band Grecia Maggiore erschienen. Dieser fokussiert ganz spezifisch die kulturellen Verflechtungen zwischen Ost und West, die Burkert so am Herzen liegen. Das sogenannte Magna Grecia, wo östliche Einflüsse bei von orphisch-bakchischen Inhalten bestimmten Figuren wie Parmenides und Empedokles wirkmächtig werden, wird durch einen konsequenten Einbezug des Vorderen Orients zu einem noch größeren Griechenland, eben zu "Grecia Maggiore", dem einheitlichen multiethnischen Kulturraum des gesamten Mittelmeergebiets. Die gegenseitigen kulturellen Vernetzungen zwischen Ost und West, nicht nur in fernen Zeiten dunkler Jahrhunderte, sondern konkret in der archaischen Epoche, stehen im Mittelpunkt. In einer sich neuen Themenfeldern öffnenden Gräzistik ist es ein lohnenswertes Projekt, die diesbezüglichen Forschungsanstöße Burkerts aus heutiger Perspektive zusammenzufassen und Revue passieren zu lassen. Antonio Panaino (2009) zeigt, wie dem Begriff magos zunächst nichts Negatives anhaftet und wie er erst in der Begegnung mit dem Fremden pejorative Konnotationen des Gauklers, Hexers und Aufschneiders erhielt. Wissenschaftlich bedeutend ist vor allem die intensive Behandlung der rituellen Kolumnen im DerveniPapyrus, zu dessen Deutung Burkert so viel beigetragen hat. Entgegen der mittlerweile erschienenen offiziellen Edition (Kouremenos/Parássoglou/ Tsantsanoglou 2006), wo zu den dort genannten magoi ein sehr einseitig negatives Bild gezeichnet und die Meinung vertreten wird, der DerveniAutor würde sich damit kritisch von ihren unwissenschaftlichen Praktiken distanzieren, legt Panaino dar, dass ihre Erwähnung durchaus das konkrete Auftreten iranischer Spezialisten im Kontakt mit Göttern implizieren könnte. Ivo Hajnal (2009) widmet sich einem weiteren Forschungsinteresse Burkerts, der Auswirkung der im achten Jahrhundert v. Chr. wie- 
derentdeckten Schriftlichkeit für die griechische Kultur. Hajnal präsentiert ein faszinierendes und sehr aktuelles Bild zur mykenischen Schriftkultur des Linear B und zu den Gründen ihres Untergangs in den sogenannten Dunklen Jahrhunderten. Er zeigt, dass die Schriftlichkeit dort nur partiell gelten kann, der Textbegriff im Vergleich zur vorderorientalischen Schriftkultur nur eingeschränkt ist und komplementär dazu den pragmatischen Kontext sowie eine mündliche Rezitation benötigt. Dies sei auch der Grund des totalen Verschwindens dieser frühen griechischen Literarizität in nuce. Selbst mit dem Aufkommen der Alphabetschrift im achten Jahrhundert v. Chr. war diese wiederum zunächst in ihrer Textlichkeit noch nicht völlig von Oralitätsmerkmalen losgelöst, konnte sich dann aber aufgrund eines panhellenischen Identitätsverständnisses und einer polyzentrischen Gesellschaft, die individuelle Kommunikation benötigt, schnell ganz verselbstständigen. Burkert (2009) selbst liefert ein wunderbares Panorama des interkulturellen Austausches zwischen Griechen und Persern von den Perserkriegen bis in das dazu bisher eher vernachlässigte vierte Jahrhundert v. Chr. Burkerts Schülerin Laura Gemelli Marciano (2009) weist anhand der Fragmente 14 und 15 DK nach, wie der frühe, in Milet wirkende Philosoph Heraklit persische rituelle Spezialisten, eben auch magoi, die dort auch real präsent waren, als Folie für seine 'griechische' Philosophie verwendet. Diese beruht freilich ebenfalls auf religiösen Ideen und Bildern, zumal Heraklit aufgrund verwandtschaftlicher Beziehungen eng mit Eleusis und dem dortigen Mysterienkult verbunden ist. Zuletzt gibt Giovanni Casadio (2009) ein durchaus kritisches Bild des geistigen Kontexts, in dem Burkert sich in seinen Forschungen bewegt, wenn er sich der Methode des ex oriente lux bedient, das heißt wenn er für griechische kulturelle Phänomene konkrete Anregungen und Vorlagen aus vorderorientalischen Kulturen auszumachen versucht.

Unser Unternehmen des Bielefelder Autorenkolloquiums (2007) hatte wenig später das ambitionierte Ziel, die Wirkung Burkerts insgesamt zu beleuchten. Auf dem beschränkten Gebiet der Gräzistik will ich die Ergebnisse nochmals wie folgt zusammenfassen: Walter Burkert ist entweder mit ganz großen Würfen, die den Menschen an sich erklären, oder mit Detailstudien hervorgetreten. Hauptsächlich ist er an der Erforschung der griechischen Religion in allen Facetten interessiert, wobei er souverän alle altertumswissenschaftlichen Teildisziplinen einbezieht. Sein ureigenes Feld, die philologisch-literaturwissenschaftliche Deutung der großen griechischen Texte, wird für ihn hingegen nur zum wichtigen Nebenschau- 
platz. Selbst im kleinsten Detail geht es ihm meist um die wichtigste Frage, um den Menschen schlechthin. Homo Necans entfaltet vor allem deswegen so große Wirkung, weil Burkert letztlich einen tragischen Weltentwurf über das Leben angesichts des Todes unternimmt. Der Aufsatz über das Opfer und die Tragödie (Burkert 1966b) liegt dem Drama des Rituals und Mythos implizit zu Grunde. In seiner Lektüre, die Lorenz, Freud, aber auch Nietzsche und W. F. Otto vereint, stößt Burkert schon zum Performativen des Rituals vor. Mit diesem anthropologisch-philosophischen Rüstzeug versucht er anhand der exemplarischen griechischen Kultur und Religion über die psycho-ethologische Verfasstheit des Menschen aufzuklären. Er zögert jedoch, die in seiner frühen Schaffensperiode erzielten Ergebnisse in größerem Stil auf die bedeutenden Texte der griechischen Literatur im Einzelnen anzuwenden. Doch dient er zugleich als Inspirationsquelle, Fundgrube und Anregung für andere Literaturwissenschaftler, die mit einer kulturwissenschaftlich geprägten Methode das mythisch-rituelle Substrat aufdecken. In der Gräzistik ist es erst seit Burkert wieder legitim, sich nach den einseitigen Auswüchsen der von den Cambridge Ritualists angestoßenen Forschungen mit Mythos und Ritual in der griechischen Literatur zu beschäftigen. $\mathrm{Zu}$ einer neuen reflektierten Methodologie kommt diese Richtung zuletzt, seitdem man systematisch die mythisch-rituelle Poetik griechischer Texte untersucht. Hier geht man der Fragestellung nach, wie Autoren im freien ästhetischen Spiel von Variation und Kombination Elemente des Mythos und des Rituals für die Schaffung von Literatur zusammenstellen. Der Stellenwert des Burkertschen Werks als literarische Heuristik bleibt freilich keineswegs auf die Klassische Philologie beschränkt, sondern erstreckt sich heute beinahe auf alle Literaturen und Zeiten. Burkerts Kultstatus und Potential als Inspirationsquelle erklärt sich wohl auch aus der Tatsache, dass von seiner eigentümlichen, nahezu poetischen Prosa der frühen Schriften bis Homo Necans, wo das Unheimliche und Unabgegoltene des Griechentums auf kongeniale Art abgehandelt wird, ein ganz besonderer Reiz ausgeht. Inhalt und Form treffen den damaligen Zeitgeist - das Hehre und Klassische wird mit seiner anderen Seite konfrontiert. Mit Burkert ist ein ganz neues Bild der Griechen entstanden. 


\section{Bibliografie}

Alexiou 2002, M.: After Antiquity. Greek Language, Myth, and Metaphor, Ithaca/London 2002.

Aronen 1992, J.: "Notes on Athenian Drama as Ritual Myth-Telling within the Cult of Dionysos", Arctos 26, 1992, 19-37.

Auffarth 1991, C.: Der drohende Untergang. 'Schöpfung' in Mythos und Ritual im Alten Orient und in Griechenland am Beispiel der Odyssee und des Ezechielbuches, Berlin/New York 1991.

Auffarth 1994, C.: 'Der Opferstreik. Ein altorientalisches 'Motiv' bei Aristophanes und im homerischen Hymnus", GB 20, 1994, 59-86.

Auffarth 1999, C.: "Ein seltsamer Priester und seine Jünger. Typisches und Charakteristisches im Bühnen-Sokrates des Aristophanes”, in: K. Pestalozzi (Hrsg.), Der fragende Sokrates (Colloquium Rauricum 6), Stuttgart/Leipzig 1999, 77-97.

Auffarth 2004, C.: "Let Women Speak in the Assembly. Symbolic Reversal in Aristophanes' Ekklesiazousai”, in: S. des Bouvrie (Hrsg.), Myth and Symbol II. Symbolic Phenomena in Ancient Greek Culture, Bergen 2004, 43-62.

Auffarth 2007, C.: "Ritual, Performanz, Theater. Die Religion der Athener in Aristophanes’ Komödien”, in: Bierl/Lämmle/Wesselmann 2007a, 387-414.

Bierl 1991, A. F. H.: Dionysos und die griechische Tragödie. Politische und 'metatheatralische' Aspekte im Text, Tübingen 1991.

Bierl 1994, A.: "Karion, die Karer und der Plutos des Aristophanes als Inszenierung eines anthesterienartigen Ausnahmefestes", in: A. Bierl und P. von Möllendorff (unter Mitwirkung von S. Vogt) (Hrsg.), Orchestra. Drama - Mythos - Bühne. Festschrift für Hellmut Flashar anläßlich seines 65. Geburtstages, Stuttgart/Leipzig 1994, 3043.

Bierl 2001, A.: Der Chor in der Alten Komödie. Ritual und Performativität (unter besonderer Berücksichtigung von Aristophanes' Thesmophoriazusen und der Phalloslieder fr. $851 P M G)$, München/Leipzig 2001.

Bierl 2002a, A.: "Experimentelle Innovation und ihre rituell-pragmatischen Grenzen in der Alten Komödie", QUCC N. F. 72.3 (101), 2002, 7-21.

Bierl 2002b, A.: “Rezension von Riu 1999”, Gnomon 74, 2002, 196-203.

Bierl 2003, A.: “'Ich aber (sage), das Schönste ist, was einer liebt!' Eine pragmatische Deutung von Sappho Fr. 16 LP/V”, QUCC N. F. 74.2 (103), 2003, 91-124.

Bierl 2004, A.: "Alt und Neu bei Aristophanes (unter besonderer Berücksichtigung der Wolken)", in: A. von Müller und J. von Ungern-Sternberg (Hrsg.), Die Wahrnehmung des Neuen in Antike und Renaissance (Colloquium Rauricum 8), München/Leipzig 2004, 1-24.

Bierl 2006, A.: "Tragödie als Spiel und das Satyrspiel. Die Geburt des griechischen Theaters aus dem Geiste des Chortanzes und seines Gottes Dionysos", in: J. Sánchez 
de Murillo und M. Thurner (Hrsg.), Aufgang. Jahrbuch für Denken, Dichten, Musik III. Kind und Spiel, Stuttgart 2006, 111-138.

Bierl 2007a, A.: "Literatur und Religion als Rito- und Mythopoetik. Überblicksartikel zu einem neuen Ansatz in der Klassischen Philologie”, in: Bierl/Lämmle/Wesselmann 2007a, 1-76.

Bierl 2007b, A.: "Mysterien der Liebe und die Initiation Jugendlicher. Literatur und Religion im griechischen Roman”, in: Bierl/Lämmle/Wesselmann 2007b, 239-334.

Bierl 2007c, A.: “Le 'chamanisme' et la comédie ancienne. Recours générique à un atavisme et guérison (avec une application à l'exemple de la Paix d'Aristophane)", Methodos 7, 2007 (http://methodos.revues.org/document625.html).

Bierl 2007d, A.: "L'uso intertestuale di Alcmane nel finale della Lisistrata di Aristofane. Coro e rito nel contesto performativo", in: F. Perusino und M. Colantonio (Hrsg.), Dalla lirica corale alla poesia drammatica. Forme e funzioni del canto corale nella tragedia e nella commedia greca, Pisa 2007, 259-290.

Bierl 2009a, A.: "Der griechische Roman - ein Mythos? Gedanken zur mythischen Dimension von Longos' Daphnis und Chloe", in: U. Dill und C. Walde (Hrsg.), Antike Mythen. Medien, Transformationen und Konstruktionen, Berlin/New York 2009, 709-739.

Bierl 2009b, A.: "Antike Mysterien - ein Weg zur Vollkommenheit und die literarische Verarbeitung in Apuleius' Metamorphosen", in: A. Assmann und J. Assmann (Hrsg.), Vollkommenheit, München 2009, 47-66 (im Druck).

Bierl 2010a, A.: "Prozessionen auf der griechischen Bühne. Performativität des einziehenden Chors als Manifestation des Dionysos in der Parodos der Euripideischen Bakchen", in: K. Gvozdeva und H. R. Velten (Hrsg.), Prozession und Medien. Texte und Bilder ritueller Bewegung in der Vormoderne. Performativité processionelle. Discours et images prémodernes du mouvement rituel, Heidelberg 2010 (im Druck).

Bierl 2010b, A.: "From Mystery to Initiation. A Mytho-Ritual Poetics of Love and Sex in the Ancient Novel - even in Apuleius' Golden Ass?" (Plenarvortrag gehalten am 22.7.2008 auf der International Conference on the Ancient Novel IV in Lissabon und auf der Konferenz-CD gespeichert; wird voraussichtlich in einem von mir hrsg. Teilband der Acta in Ancient Narrative 2010 publiziert).

Bierl (in Vorbereitung), A.: "Empedokles: Philosoph, Arzt, Dichter und religiöser Prophet. Vom Mythos zum Logos?", Antrittsvorlesung, Basel 13.5.2003.

Bierl/Lämmle/Wesselmann 2007a, A./R./K. (Hrsg.): Literatur und Religion I. Wege zu einer mythisch-rituellen Poetik bei den Griechen, Berlin/New York 2007.

Bierl/Lämmle/Wesselmann 2007b, A./R./K. (Hrsg.): Literatur und Religion II. Wege zu einer mythisch-rituellen Poetik bei den Griechen, Berlin/New York 2007.

Bowie 1993, A. M.: Aristophanes. Myth, Ritual and Comedy, Cambridge 1993.

Braungart 1996, W.: Ritual und Literatur, Tübingen 1996.

Braungart 2007, W.: "Mythos und Ritual, Leiden und Opfer. Ein strukturgeschichtlicher Versuch zur Tragödie”, in: Bierl/Lämmle/Wesselmann 2007b, 359-423. 
Brelich 1969, A.: Paides e Parthenoi, I, Rom 1969.

Brelich 1975, A.: “Aristofane. Commedia e religione”, in: M. Detienne (Hrsg.), Il mito. Guida storica e critica, Roma/Bari $1982^{3}\left(1975^{1}\right), 103-118$.

Bremmer 1983, J.: The Early Greek Concept of the Soul, Princeton 1983.

Burkert 1955, W.: Zum altgriechischen Mitleidsbegriff, Diss. Erlangen 1955.

Burkert 1960, W.: "Das Lied von Ares und Aphrodite. Zum Verhältnis von Odyssee und Ilias", RhM 103, 1960, 130-144.

Burkert 1962a, W.: Weisheit und Wissenschaft. Studien zu Pythagoras, Philolaos und Platon, Nürnberg 1962 (engl. Fassung: Lore and Science in Ancient Pythagoreanism, translated by E. L. Minar, Jr., Cambridge, MA 1972).

Burkert 1962b, W.: “Гóns. Zum griechischen 'Schamanismus”, RhM 105, 1962, 36-55.

Burkert 1965, W.: "Demaratos, Astrabakos und Herakles. Königsmythos und Politik zur Zeit der Perserkriege (Herodot 6, 67-69)", $M H$ 22, 1965, 166-177.

Burkert 1966a, W.: "Kekropidensage und Arrhephoria. Vom Initiationsritus zum Panathenäenfest”, Hermes 94, 1966, 1-25 (Nachdruck in: Burkert 1990a, 40-59).

Burkert 1966b, W.: “Greek Tragedy and Sacrificial Ritual”, GRBS 7, 1966, 87-121 (dt. Fassung in: Burkert 1990a, 13-39).

Burkert 1968, W.: "Orpheus und die Vorsokratiker. Bemerkungen zum Derveni-Papyrus und zur pythagoreischen Zahlenlehre", $A \& A$ 14, 1968, 93-114.

Burkert 1969, W.: "Das Proömium des Parmenides und die Katabasis des Pythagoras", Phronesis 14, 1969, 1-30.

Burkert 1970, W.: "Iason, Hypsipyle, and New Fire at Lemnos. A Study in Myth and Ritual", CQ 20, 1970, 1-16 (dt. Fassung in: Burkert 1990a, 60-76).

Burkert 1972, W.: Homo Necans. Interpretationen altgriechischer Opferriten und Mythen, Berlin/New York $1997^{2}\left(1972^{1}\right)$ (engl. Fassung: Homo Necans. The Anthropology of Ancient Greek Sacrificial Ritual and Myth, translated by P. Bing, Berkeley et al. 1983).

Burkert 1973, W.: "Von Amenophis II. zur Bogenprobe des Odysseus", GB 1, 1973, 6978.

Burkert 1974, W.: “Die Absurdität der Gewalt und das Ende der Tragödie. Euripides' Orestes", A\&A 20, 1974, 97-109.

Burkert 1975, W.: “Apellai und Apollon”, RhM 118, 1975, 1-21.

Burkert 1976, W.: "Das hunderttorige Theben und die Datierung der Ilias", WS 89 (N. F. 10), 1976, 5-21.

Burkert 1977, W.: Griechische Religion der archaischen und klassischen Epoche, Stuttgart et al. 1977 (engl. Fassung: Greek Religion. Archaic and Classical, translated by J. Raffan, Cambridge, MA 1985).

Burkert 1979, W.: Structure and History in Greek Mythology and Ritual, Berkeley et al. 1979. 
Burkert 1981, W.: "Seven against Thebes. An Oral Tradition between Babylonian Magic and Greek Literature", in: C. Brillante, M. Cantilena und C. O. Pavese (Hrsg.), I poemi epici rapsodici non omerici e la tradizione orale (Atti del convegno di Venezia, 28-30 settembre 1977), Padova 1981, 29-48.

Burkert 1984a, W.: Anthropologie des religiösen Opfers. Die Sakralisierung der Gewalt (Privatdruck: Carl Friedrich von Siemens Stiftung - Themen 40), München $1987^{2}$ $\left(1984^{1}\right)$.

Burkert 1984b, W.: Die orientalisierende Epoche in der griechischen Religion und Literatur (Sitzungsberichte der Heidelberger Akademie der Wissenschaften, Philosophisch-historische Klasse 1984.1), Heidelberg 1984 (engl. Fassung: The Orientalizing Revolution. Near Eastern Influence on Greek Culture in the Early Archaic Age, translated by M. E. Pinder and W. Burkert, Cambridge, MA/London 1992).

Burkert 1984c, W.: “Sacrificio-sacrilegio. Il 'trickster' fondatore”, StudStor 25, 1984, 835-845.

Burkert 1985a, W.: "Opferritual bei Sophokles. Pragmatik-Symbolik-Theater”, AU 28.2, 1985, 5-20.

Burkert 1985b, W.: "Das Ende des Kroisos. Vorstufen einer herodoteischen Geschichtserzählung”, in: Catalepton. Festschrift für Bernhard Wyss zum 80. Geburtstag, Basel 1985, 4-15.

Burkert 1987a, W.: “The Problem of Ritual Killing”, in: R. G. Hamerton-Kelly (Hrsg.), Violent Origins. Walter Burkert, René Girard, and Jonathan Z. Smith on Ritual Killing and Cultural Formation, Stanford 1987, 149-176.

Burkert 1987b, W.: Ancient Mystery Cults, Cambridge, MA/London 1987 (dt. Fassung: Antike Mysterien. Funktionen und Gehalt, München $\left.2003^{4}\left[1990^{1}\right]\right)$.

Burkert 1990a, W.: Wilder Ursprung. Opferritual und Mythos bei den Griechen, Berlin 1990.

Burkert 1990b, W.: “Ein Datum für Euripides' Elektra: Dionysia 420 v. Chr.”, MH 47, 1990, 65-69.

Burkert 1991, W.: "Oedipus, Oracles, and Meaning. From Sophocles to Umberto Eco", in: The Samuel James Stubbs Lecture Series 1, Toronto 1991.

Burkert 1993a, W.: Platon in Nahaufnahme. Ein Buch aus Herculaneum (Lectio Teubneriana 2), Stuttgart/Leipzig 1993.

Burkert 1993b, W.: "Attische Feste in der 'Aulischen Iphigenie' des Euripides”, in: J. Dalfen, G. Petersmann und F. F. Schwarz (Hrsg.), Religio Graeco-Romana. Festschrift für Walter Pötscher, Graz 1993, 87-92.

Burkert 1996a, W.: Creation of the Sacred. Tracks of Biology in Early Religions, Cambridge MA/London 1996 (dt. Fassung: Kulte des Altertums. Biologische Grundlagen der Religion, München 1998).

Burkert 1996b, W.: Klassisches Altertum und antikes Christentum. Probleme einer übergreifenden Religionswissenschaft, Berlin/New York 1996.

Burkert 1997a, W.: "Nachwort 1996”, in: Burkert 1972 (= 1997²), 333-352. 
Burkert 1997b, W.: "Star Wars or One Stable World? A Problem of Presocratic Cosmogony (PDerv. Col. XXV)", in: A. Laks und G. W. Most (Hrsg.), Studies on the Derveni Papyrus, Oxford 1997, 167-174.

Burkert 1999a, W.: Da Omero ai Magi. La tradizione orientale nella cultura greca, Venedig 1999 (erweiterte dt. Fassung: Die Griechen und der Orient. Von Homer bis zu den Magiern, München 2003).

Burkert 1999b, W.: "The Logic of Cosmogony”, in: Buxton 1999, 87-106.

Burkert 1999c, W.: "Von Selinus zu Aischylos. 'Reinigung' im Ritual und im Theater", in: Berlin-Brandenburgische Akademie der Wissenschaften, Berichte und Abhandlungen 7, Berlin 1999, 23-38.

Burkert 2000, W.: "Mythen um Oedipus. Familienkatastrophe und Orakelsinn", in: Freiburger Universitätsblätter 148.2, Freiburg 2000, 7-20.

Burkert 2001, W.: Kleine Schriften I. Homerica, hrsg. von C. Riedweg, Göttingen 2001.

Burkert 2003a, W.: Die Griechen und der Orient. Von Homer bis zu den Magiern, München 2003 (ital. Originalfassung: Burkert 1999a).

Burkert 2003b, W.: Kleine Schriften II. Orientalia, hrsg. von L. Gemelli Marciano, Göttingen 2003.

Burkert 2004, W.: "Initiation", in: Thesaurus cultus et rituum antiquorum, II, Los Angeles 2004, 91-124.

Burkert 2006, W.: Kleine Schriften III. Mystica, Orphica, Pythagorica, hrsg. von F. Graf, Göttingen 2006.

Burkert 2007, W.: Kleine Schriften VII. Tragica et Historica, hrsg. von W. Rösler, Göttingen 2007.

Burkert 2008, W.: Kleine Schriften VIII. Philosophica, hrsg. von T. A. Szlezák und K.-H. Stanzel, Göttingen 2008.

Burkert 2009, W.: "Im Schatten des Basileus. Griechisch-persische Kulturbegegnungen", in: Riedweg 2009, 87-97.

Buxton 1999, R. (Hrsg.): From Myth to Reason? Studies in the Development of Greek Thought, Oxford 1999.

Calame 2000, C.: Poétique des mythes dans la Grèce antique, Paris 2000.

Calame 2003, C.: Myth and History in Ancient Greece. The Symbolic Creation of a Colony, Princeton/Oxford 2003.

Casadio 2009, G.: "Ex oriente lux?", in: Riedweg 2009, 123-161.

Cerri 1999, G.: "La poesia di Parmenide", QUCC N. F. 63.3 (92), 1999, 7-27.

Chiasson 2005, C. C.: "Myth, Ritual, and Authorial Control in Herodotus' Story of Cleobis and Biton (Hist. 1. 31)", AJPh 126, 2005, 41-64.

Cook 1995, E. F.: The Odyssey in Athens. Myths of Cultural Origins, Ithaca/London 1995.

Cornford 1907, F. M.: Thucydides Mythistoricus, London 1907 (Nachdruck 1965). 
Cornford 1914, F. M.: The Origin of Attic Comedy, ed. by J. Henderson, Ann Arbor 1993 (Cambridge 1914 ${ }^{1}$, Garden City, NY $1961^{2}$ ).

des Bouvrie 1993, S.: "Creative Euphoria. Dionysos and the Theatre", Kernos 6, 1993, 79-112.

Dodds 1951, E. R.: The Greeks and the Irrational, Berkeley 1951 (dt. Fassung: Die Griechen und das Irrationale, übers. von H.-J. Dirksen, Darmstadt $1991^{2}$ ).

Farioli 2001, M.: Mundus alter. Utopie e distopie nella commedia greca antica, Mailand 2001.

Foley 1985, H. P.: Ritual Irony. Poetry and Sacrifice in Euripides, Ithaca 1985.

Gemelli Marciano 2007, M. L.: "Einführung”, in: Die Vorsokratiker, I, hrsg. von M. L. Gemelli Marciano, Düsseldorf 2007, 373-465.

Gemelli Marciano 2008, M. L.: “Images and Experience. At the Roots of Parmenides' Aletheia", AncPh 28, 2008, 21-48.

Gemelli Marciano 2009, “A chi profetizza Eraclito di Efeso? Eraclito 'specialista del sacro' fra oriente e occidente”, in: Riedweg 2009, 99-122.

Gibert 2003, J.: “Apollo's Sacrifice. The Limits of a Metaphor in Greek Tragedy”, HSCPh 101, 2003, 159-206.

Graf 1987, F.: “Orpheus: A Poet Among Men”, in: J. Bremmer (Hrsg.), Interpretations of Greek Mythology, London/Sydney 1987, 80-106.

Graf 1991, F.: "Kultur als Macht und Schutzmacht. Zum wissenschaftlichen Werk von Walter Burkert", NZZ 21, 26./27. Jan. 1991, 69.

Graf 1998, F. (Hrsg.): Ansichten griechischer Rituale. Geburtstags-Symposium für Walter Burkert, Stuttgart/Leipzig 1998.

Guidorizzi 1996, G. (Hrsg.): Aristofane. Le Nuvole, Milano 1996.

Hajnal 2009, I.: "Wort und Schrift in der mykenischen Bronzezeit. Mit- oder Nebeneinander?", in: Riedweg 2009, 55-86.

Harth/Schenk 2004, D./G. J. (Hrsg.): Ritualdynamik. Kulturübergreifende Studien zur Theorie und Geschichte rituellen Handelns, Heidelberg 2004.

Henrichs 2000, A.: "Drama and Dromena. Bloodshed, Violence, and Sacrificial Metaphor in Euripides", HSCPh 100, 2000, 173-188.

Henrichs 2004, A.: “'Let the Good Prevail'. Perversions of the Ritual Process in Greek Tragedy”, in: Yatromanolakis/Roilos 2004, 189-198.

Henrichs 2006, A.: "Blutvergießen am Altar. Zur Ritualisierung der Gewalt im griechischen Opferkult”, in: B. Seidensticker und M. Vöhler (Hrsg.), Gewalt und Ästhetik. Zur Gewalt und ihrer Darstellung in der griechischen Klassik, Berlin/New York 2006, 59-87.

Hoffman 1989, R. J.: "Ritual License and the Cult of Dionysus", Athenaeum N. F. 67, 1989, 91-115.

Hofmann 1976, H.: Mythos und Komödie. Untersuchungen zu den Vögeln des Aristophanes, Hildesheim/New York 1976. 
Isler-Kerényi 2001, C.: Dionysos nella Grecia arcaica. Il contributo delle immagini, Pisa/Rom 2001.

Janka/Schäfer 2002, M./C. (Hrsg.): Platon als Mythologe. Neue Interpretationen zu den Mythen in Platons Dialogen, Darmstadt 2002.

Kingsley 1995, P.: Ancient Philosophy, Mystery, and Magic. Empedocles and Pythagorean Tradition, Oxford 1995.

Kingsley 2000, P.: Die Traumfahrt des Parmenides. Die mystischen Wurzeln der westlichen Zivilisation, aus dem Engl. von K. Rausch, Frankfurt a. M. 2000 (engl. Originalfassung: In the Dark Places of Wisdom, Inverness, CA 1999).

Kingsley 2003, P.: Reality, Inverness, CA 2003.

Kouremenos/Parássoglou/Tsantsanoglou 2006, T./G. M./K. (Hrsg.): The Derveni Papyrus, Florenz 2006.

Krummen 1990, E.: Pyrsos Hymnon. Festliche Gegenwart und mythisch-rituelle Tradition als Voraussetzung einer Pindarinterpretation (Isthmie 4, Pythie 5, Olympie 1 und 3), Berlin/New York 1990.

Krummen 1998, E.: "Ritual und Katastrophe. Rituelle Handlung und Bildersprache bei Sophokles und Euripides”, in: Graf 1998, 296-325.

Krummen 2002, E.: "Sokrates und die Götterbilder. Zur Erkenntnis der höchsten Ideen in Platons Symposion (215 ab)", Perspektiven der Philosophie. Neues Jahrbuch 28, 2002, 11-45.

Krummen 2007, E.: “'Schön nämlich ist das Wagnis'. Rituelle Handlung und mythische Erzählung in Platons Phaidon”, in: Bierl/Lämmle/Wesselmann 2007b, 91-139.

Lada-Richards 1999, I.: Initiating Dionysus. Ritual and Theatre in Aristophanes' Frogs, Oxford 1999.

Lämmle 2007, R.: "Der eingeschlossene Dritte. Zur Funktion des Dionysos im Satyrspiel”, in: Bierl/Lämmle/Wesselmann 2007a, 335-386.

Lord 1960, A. B.: The Singer of Tales, Cambridge, MA 1960.

Martin 1987, R. P.: "Fire on the Mountain. Lysistrata and the Lemnian Women", CA 6, 1987, 77-105.

Merkelbach 1962, R.: Roman und Mysterium in der Antike, München 1962.

Merkelbach 1988, R.: Die Hirten des Dionysos. Die Dionysos-Mysterien der römischen Kaiserzeit und der bukolische Roman des Longus, Stuttgart 1988.

Merkelbach 1995, R.: Isis Regina - Zeus Sarapis. Die griechisch-ägyptische Religion nach den Quellen dargestellt, Stuttgart/Leipzig $2001^{2}\left(1995^{1}\right)$.

Meuli 1935, K.: "Scythica", Hermes 70, 1935, 121-176 (Nachdruck mit Ergänzungen in: Gesammelte Schriften, II, hrsg. von T. Gelzer, Basel/Stuttgart 1975, 817-879).

Morgan 2000, K. A.: Myth and Philosophy from the Presocratics to Plato, Cambridge 2000.

Most 1990, G.: "Strenge Erforschung wilder Ursprünge. Walter Burkert über Mythos und Ritus", Vorwort zu Burkert 1990a, 7-12. 
Münz 1998, R.: Theatralität und Theater. Zur Historiographie von Theatralitätsgefügen, hrsg. von G. Amm, Berlin 1998.

Murray 1999, P.: "What Is a Mythos for Plato?", in: Buxton 1999, 251-262.

Nagy 1990, G.: Pindar's Homer. The Lyric Possession of an Epic Past, Baltimore/London 1990.

Nagy 2007, G.: "Did Sappho and Alcaeus Ever Meet? Symmetries of Myth and Ritual in Performing the Songs of Ancient Lesbos", in: Bierl/Lämmle/Wesselmann 2007a, 211-269.

Panaino 2009, A.: “Aspetti della complessità degli influssi interculturali tra Grecia ed Iran”, in: Riedweg 2009, 19-53.

Papadopoulou-Belmehdi 1994, I.: Le chant de Pénélope. Poétique du tissage féminin dans l'Odyssée, Paris 1994.

Parry 1928, M.: L'épithète traditionelle dans Homére. Essai sur un problème de style homérique, Paris 1928.

Parry 1971, M.: The Making of Homeric Verse. The Collected Papers of Milman Parry, ed. by A. Parry, Oxford 1971.

Peirce 1993, S.: "Death, Revelry, and Thysia", CA 12, 1993, 219-266 und Abb. 1-18.

Pucci 1977, P.: "Euripides. The Monument and the Sacrifice", Arethusa 10, 1977, 165195.

Riedweg 1987, C.: Mysterienterminologie bei Platon, Philon und Klemens von Alexandrien, Berlin/New York 1987.

Riedweg 1995, C.: "Orphisches bei Empedokles", A\&A 41, 1995, 34-59.

Riedweg 2009, C. (Hrsg.): Grecia maggiore. Intrecci culturali con l'Asia nel periodo arcaico (Simposio in occasione del $75^{\circ}$ compleanno di Walter Burkert), Basel 2009.

Riu 1999, X.: Dionysism and Comedy, Lanham et al. 1999.

Schefer 2000, C.: “'Nur für Eingeweihte!'. Heraklit und die Mysterien”, A\&A 46, 2000, 46-75.

Seaford 1981, R.: "Dionysiac Drama and the Dionysiac Mysteries", CQ 31, 1981, 252 275.

Seaford 1984, R. A. S.: Euripides. Cyclops, edited with introduction and commentary, Oxford $1988^{2}\left(1984^{1}\right)$.

Seaford 1986, R.: "Immortality, Salvation, and the Elements", HSCPh 90, 1986, 1-26.

Seaford 1993, R.: "Dionysus as Destroyer of the Household. Homer, Tragedy, and the Polis", in: T. H. Carpenter und C. A. Faraone (Hrsg.), Masks of Dionysus, Ithaca/London 1993, 115-146.

Seaford 1994, R.: Reciprocity and Ritual. Homer and Tragedy in the Developing CityState, Oxford 1994.

Seaford 1996, R.: Euripides. Bacchae, with an introduction, translation and commentary, Warminster 1996. 
Segal 1982, C.: Dionysiac Poetics and Euripides' Bacchae, expanded edition with a new afterword by the author, Princeton $1997^{2}\left(1982^{1}\right)$.

Seidensticker 1979, B.: "Sacrificial Ritual in the Bacchae", in: G. W. Bowersock, W. Burkert und M. C. J. Putnam (Hrsg.), Arktouros. Hellenic Studies Presented to Bernard M. W. Knox on the Occasion of his 65th Birthday, Berlin/New York 1979, 181190.

Sfyroeras 2004, P.: "From Sacrifice to Feast. A Ritual Pattern in Aristophanic Comedy", in: D. L. Cairns und R. A. Knox (Hrsg.), Law, Rhetoric, and Comedy in Classical Athens. Essays in Honour of Douglas M. MacDowell, London/Swansea 2004, 251268.

Stadter 2004, P. A.: "From the Mythical to the Historical Paradigm. The Transformation of Myth in Herodotus", in: J. M. Candau Morón, F. J. González Ponce und G. Cruz Andreotti (Hrsg.), Historia y mito. El pasado legendario como fuente de autoridad, Malaga 2004, 31-46.

Vernant 1972, J.-P.: “Ambiguité et renversement. Sur la structure énigmatique d' 'Edipe Roi'”, in: J.-P. Vernant und P. Vidal-Naquet (Hrsg.), Mythe et tragédie en Grèce ancienne, I, Paris $1986^{3}\left(1972^{1}\right), 99-131$.

Versnel 1993, H. S.: Inconsistencies in Greek and Roman Religion II. Transition and Reversal in Myth and Ritual, Leiden et al. 1993.

Vidal-Naquet 1969, P.: "Chasse et sacrifice dans l'Orestie d'Eschyle", PdP 129, 1969, 401-425 (Nachdruck in: J.-P. Vernant und P. Vidal-Naquet (Hrsg.), Mythe et tragédie en Grèce ancienne, I, Paris $1986^{3}$ [1972 ${ }^{1}$, 133-158).

Wesselmann 2007, K.: "Xerxes und die Frau des Masistes (Hdt. 9.108-113). Mythische Erzählstruktur in Herodots Historien”, in: Bierl/Lämmle/Wesselmann 2007b, 1-39.

West 1983, M. L.: The Orphic Poems, Oxford 1983.

Wolff 1992, C.: "Euripides' Iphigenia among the Taurians. Aetiology, Ritual, and Myth", CA 11, 1992, 308-334.

Yatromanolakis 2003, D.: "Ritual Poetics in Archaic Lesbos. Contextualizing Genre in Sappho", in: Yatromanolakis/Roilos 2003, 43-59.

Yatromanolakis 2007, D.: Sappho in the Making. The Early Reception, Washington 2007.

Yatromanolakis/Roilos 2003, D./P.: Towards a Ritual Poetics, Athen 2003.

Yatromanolakis/Roilos 2004, D./P. (Hrsg.): Greek Ritual Poetics, Cambridge, MA 2004.

Zannini Quirini 1987, B.: Nephelokokkygia. La prospettiva mitica degli Uccelli di Aristofane, Rom 1987.

Zeitlin 1965, F. I.: "The Motif of the Corrupted Sacrifice in Aeschylus' Oresteia", TAPhA 96, 1965, 463-508.

Zeitlin 1966, F. I.: "Postscript to Sacrificial Imaginary in the Oresteia (Ag. 1235-37)", TAPhA 97, 1966, 645-653.

Zhmud 1997, L.: Wissenschaft, Philosophie und Religion im frühen Pythagoreismus, Berlin 1997. 